Salman A. Ansari · Nathan Phillips .

Graham Stabler · Peter C. Wilkins · Rafał

Żbikowski · Kevin Knowles

\title{
Experimental Investigation of Some Aspects of Insect-like Flapping Flight Aerodynamics for Application to Micro Air Vehicles
}

Received: date / Accepted: date

\begin{abstract}
Insect-like flapping flight offers a power-efficient and highly-manoeuvrable basis for micro air vehicles for indoor applications. Some aspects of the aerodynamics associated with the sweeping phase of insect wing kinematics are examined by making particle image velocimetry measurements on a rotating onebladed propeller immersed in a tank of seeded water. The work is motivated by the paucity of data with quantified error on insect-like flapping flight, and aims to fill this gap by providing a detailed description of the experimental setup, quantifying the uncertainties in the measurements and explaining the results. The experiments are carried out at two Reynolds numbers-500 and 15000-accounting for scales pertaining to insects and flapping-wing micro air vehicles, respectively. Results from the experiments are used to describe prominent flow features, and Reynolds number-related differences are highlighted. In particular, the behaviour of the leading-edge vortex at these Reynolds numbers is studied and the presence of Kelvin-Helmholtz instability observed at the higher Reynolds number in computational fluid dynamics calculations is also verified.
\end{abstract}

Keywords low Reynolds number flow $\cdot$ insect flight $\cdot$ flapping wing $\cdot$ micro air vehicles $\cdot$ particle image velocimetry $\cdot$ leading-edge vortex $\cdot$ Kelvin-Helmholtz instability

The authors acknowledge the partial support of the EPSRC through grant no. EP/C535278/1 (Stabler), the ORS Award Scheme (Phillips) and the Cranfield University, Department of Aerospace, Power \& Sensors scholarships (Wilkins \& Phillips) for this work.

S. A. Ansari (凶), N. Phillips, G. Stabler, P. C. Wilkins, R. Żbikowski, K. Knowles

Cranfield University

Defence Academy of the UK, Shrivenham

Swindon SN6 8LA, UK

Tel: +44 (0)1793 785348

Fax: +44 (0)1793 785902

E-mail: s.a.ansari@cranfield.ac.uk

Published by Springer. This is the Author Accepted Manuscript. This article may be used for personal use only. The final published version (version of record) is available online at DOI:10.1007/s00348-009-0661-2.

Please refer to any applicable publisher terms of use. 


\section{Introduction}

Agile flight inside buildings, caves and tunnels is of significant military and civilian value because current surveillance assets (such as satellites or unmanned air vehicles) possess virtually no capabilities of information-gathering in enclosed spaces. The focus on indoor flight leads to the requirement of a distinct flight envelope. In addition, autonomy is required to enable mission-completion without the assistance of a human telepilot; this requires precise flight control. Current unmanned aerial vehicles (UAVs) are too large to achieve indoor flight and our research has concluded (Żbikowski, 1999a,b, 2000; Ansari et al, 2006a) that insectlike flapping flight is the optimum way to fulfil this capability - fixed wing aircraft do not have the required low-speed agility and miniature helicopters are too inefficient and noisy. Insect flapping flight, on the other hand, has been present in nature for over 300 million years (Dudley, 1992) and has been perfected over this time. Insects fly at low speeds, are extremely manoeuvrable, virtually silent and most are capable of hover. In addition, insect flapping flight offers significantly better power efficiency, particularly at low flight speeds, than both fixed-wing aircraft and rotorcraft (Woods et al, 2001; Żbikowski et al, 2003), making it ideal for our focus on flapping-wing micro air vehicles (FMAVs) for indoor flight.

FMAVs will implement insect-like wing kinematics for flight, propulsion and control. It is, therefore, of great interest to understand such kinematics and their effects in the flows they produce. In this paper, the flow around a constant-speed propeller at high angle of attack (emulating an insect-like flapping wing in the sweeping phase of its cycle) is studied experimentally. The inspiration for these experiments is drawn from the computational fluid dynamics (CFD) investigations of Wilkins et al. (Wilkins et al, 2006; Wilkins and Knowles, 2007, 2008) who performed such studies at various Reynolds numbers $(R e)$ and described their findings in terms of the development of the leading-edge vortex and occurrence of Kelvin-Helmholtz instabilities (see Section 1.2 below).

This paper is organised as follows. Insect wing kinematics and the associated aerodynamics are described in brief below, with emphasis on the leading-edge vortex and Kelvin-Helmholtz instability, followed by the aims and objectives of the study in Section 2. Section 3 describes in detail the experimental setup, noting the equipment used and explaining the methodology employed. In Section 4, possible sources of error in the experiments are identified and the uncertainty they introduce is quantified. The results from the study are discussed in detail in Section 5 and the main findings from the study are summarised in Section 6.

\subsection{Insect wing kinematics}

Insects fly by beating their wings in a manner similar to the sculling motion of the oars on a rowing boat. The kinematics described here are for Diptera, which are two-winged flies, as they are easy to analyse and emulate, and are excellent flyers. A complete flapping cycle is made up of two half-strokes-downstroke and upstroke - and two stroke reversals (see Figure 1). The downstroke describes the motion of the wing as it sweeps from its rearmost to its foremost position, relative to the insect body. The upstroke describes the return cycle. During the 
stroke reversals, the wing pitches up rapidly and flips over for the subsequent half-stroke so that the leading edge always leads. Flapping frequency is generally in the range $5-200 \mathrm{~Hz}$.
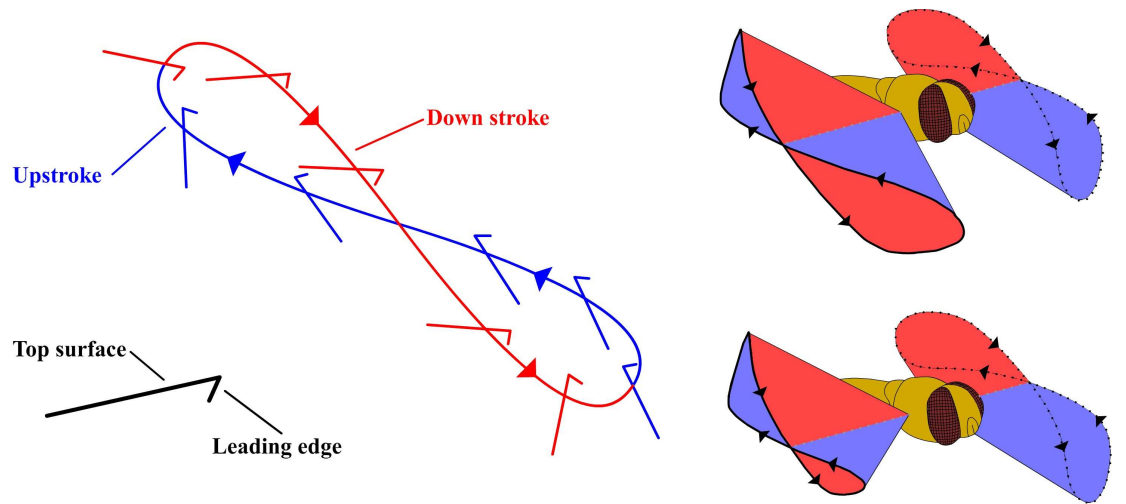

Fig. 1 Generic insect-like flapping-wing kinematics showing symmetric (right top) and asymmetric (right bottom) wing motion; colour coding: downstroke (red), upstroke (blue)

In this paper, we address some aspects associated with the flow during the sweeping phase of the flapping cycle. Wing sweep typically lasts about $80-90 \%$ of the flapping cycle (Ellington, 1984a) during which pitch changes are minimal. During a half-stroke, the wing accelerates to a roughly constant speed around the middle of the half-stroke, before slowing down to rest at the end of it. Hence, the velocity during the wing-beat cycle is non-uniform and for hover, in particular, the motion of the wing tip does not differ dramatically from a pure sinusoid (Ellington, 1984a). The insect wing normally sweeps stroke lengths typically of the order of 3-5 wing chords (Ellington, 1984b). The corresponding sweep angles (see Figure 5) are of the order of $90-120^{\circ}$, although angles greater than $180^{\circ}$ are not unknown (Thomas, priv. comm.).

\subsection{Insect-like flapping flight aerodynamics}

The aerodynamics of insect flapping flight is too extensive a subject to be addressed in great detail here. The non-uniform nature of insect-wing kinematics drives some salient flow features not expected from conventional aerodynamics. Amongst these are the Wagner effect, the Kramer effect, 'wake capture', effects due to apparent mass and the leading-edge vortex. Full details on these can be found in Ansari et al (2006a). The leading-edge vortex has been found to be of great significance in insect flight and is considered in more detail below.

Recent CFD studies by the authors have revealed the presence of KelvinHelmholtz instability not at the low Reynolds numbers pertaining to insect flight but at the higher Reynolds numbers associated with flapping-wing micro air vehicles. Hence, a discussion on Kelvin-Helmholtz instability is also included below. 
Leading-edge vortex Although it had been observed in earlier experiments (Martin and Carpenter, 1977; Maxworthy, 1979; Brodsky, 1991; Dickinson and Götz, 1993; Sunada et al, 1993) it was not until the work of Ellington et al (1996) on a scaled-up model of the hawkmoth Manduca sexta $(R e=5000$, based on mean chord and rms wing tip speed) that the significance of the leading-edge vortex for insect flapping flight received proper recognition. Later experiments by Ellington and co-workers have further corroborated its significance (Willmott et al, 1997; van den Berg and Ellington, 1997b,a).

Ellington et al (1996) reported that the leading-edge vortex, which persisted through each half-stroke, existed on the wings and proposed that it was responsible for the augmented lift forces. The overall structure of the leading-edge vortex has been likened to that observed on low-aspect-ratio delta wings (Martin and Carpenter, 1977; van den Berg and Ellington, 1997a); it is produced and fed by a leading-edge separation. Birch and Dickinson (2001) suggested that spanwise transport of the leading-edge vorticity served to remove energy from it and, hence, limited its growth and shedding (see also Rossow, 1978; Riddle et al, 1999). The leading-edge vortex starts close to the wing root and spirals towards the tip where it coalesces with the tip vortex and convects into the trailing wake (Ellington et al, 1996). It remains attached to the wing for most of the half-stroke and is shed at the end when the wing rapidly rotates in pitch. This is in contrast to the behaviour of a leading-edge vortex on a 2D wing, where regular break-away and re-formation occur Wilkins et al (2006).

Willmott et al (1997) speculated that the leading-edge vortex was formed either due to the rotational motions prior to translation or via a dynamic stall mechanism. Although dynamic stall has been suggested by others (e.g. Ellington, 1995; Dickinson, 1996), this is unlikely (Żbikowski, 2002) since a dynamic-stall vortex breaks away almost immediately and rapidly convects as soon as the wing translates (McCroskey, 1981). A detailed account of the nature of the leading-edge vortex and its possible origins can be found in Ansari (2004).

Shyy and Liu (2007) attempted to shed light on the controversy regarding the stability of the leading-edge vortex. They used a 3D RANS CFD model to compare the flows around a thrip wing at $R e=10$, a fruit fly wing at $R e=120$, and a hawkmoth wing at $R e=6000$. Their results indicated that although a leading-edge vortex was formed for each case, the detailed phenomenology of the leading-edge vortex changed as Reynolds number was increased. However, it is diffcult to draw any definite conclusions from their work because when $R e$ was changed, the wing planform was also changed. It is, therefore, not possible to accurately isolate the effect of increasing $R e$.

Whereas the leading-edge vortex may be common to most insects (Brodsky, 1991; Grodnitsky and Morozov, 1993; Willmott et al, 1997; Birch and Dickinson, 2001), its spanwise flow characteristic seems to vary with insect size (and hence Reynolds number). Recent experiments by Dickinson and co-workers (Birch and Dickinson, 2001; Sane and Dickinson, 2001; Birch et al, 2004) on a dynamicallyscaled mechanical model of the tiny fruit fly Drosophila melanogaster $(\operatorname{Re} \sim 200)$ revealed the presence of a strong, bound, leading-edge vortex but they reported only weak spanwise spiralling (unlike for the hawkmoth observed by Ellington et al (1996)), prompting them to conclude that the precise flow structure of the leading-edge vortex depends critically on Reynolds number (Birch et al, 2004). 
This is supported by Ellington (2006) who suggested that spanwise flow exists at all relevant speeds but its spiralling nature becomes less discernable as the Reynolds number decreases. Particle image velocimetry measurements by Ramasamy and Leishman (2006) on a flapping wing $(R e=15500$, based on maximum stroke velocity and mean aerodynamic chord) revealed spanwise flow within the leading-edge vortex of the same order of magnitude as the tip velocity. They also reported that the leading-edge vortex eventually broke away from the wing, but Wilkins (2008) suggests that this may have been due to wing incidence fluctuations in their experiments.

Kelvin-Helmholtz instability Kelvin-Helmholtz instability is the process whereby a vortex sheet 'bunches' into smaller areas of vorticity. It can occur anywhere where there is a shear layer within the fluid or between two different fluids, i.e. a discontinuity in velocity, density, or viscosity. The susceptibility of a vortex sheet to Kelvin-Helmholtz instability depends on three factors: the ratio of densities of the fluids on either side of the sheet, the ratio of viscosities of the fluids on either side of the sheet, and the gradient of tangential velocity of the fluids across the sheet (for further details, see Drazin and Reid, 1981). The formation of waves in water is attributed to Kelvin-Helmholtz instability. Kelvin-Helmholtz instability has also been observed in cloud formations (Drazin and Reid, 1981, p. 21). Two-dimensional flows are particularly susceptible to this type of instability (Kim et al, 2003).

The occurrence of Kelvin-Helmholtz instability also appears to increase with Reynolds number, i.e. by increasing fluid density or chord length, or by decreasing fluid viscosity. At higher Reynolds numbers, the viscous forces are lower in proportion to the inertial forces, so that vortices develop and strengthen more readily, and velocity gradients are greater. Wilkins (2008) has shown through CFD calculations that it is the gradient of velocity across a vortex sheet and not its magnitude that promotes Kelvin-Helmholtz instability. The interested reader is referred to Wilkins (2008) for a detailed discussion on the subject.

Recent CFD calculations by Wilkins and co-workers (Wilkins et al, 2006; Wilkins and Knowles, 2007) have shown Kelvin-Helmholtz instability not only for 2-D flows but also for 3-D ones. They report that Kelvin-Helmholtz instability occurs in both leading- and trailing-edge vortex sheets at higher Reynolds numbers $(\operatorname{Re}>1000)$ for both two- and three-dimensional flows, but does not result in the three-dimensional leading-edge vortex detaching from the wing (Wilkins and Knowles, 2007).

\section{Aims and Objectives}

The aim of the present experiment was to study the flow associated with the sweeping phase of insect-like flapping flight at scales relating to actual insects and to flapping-wing micro air vehicles. The intention was also to identify the behaviour of the leading-edge vortex and clarify the disparity that exists in the literature concerning it. Recent CFD studies by the authors have shown the existence of Kelvin-Helmholtz instability for the 3-D flow around a rotating propeller; a further aim of this study was to validate this finding. 


\section{Experimental Setup}

The experiments were carried out on a propeller in a water tank and flow measurements were made using a particle image velocimetry (PIV) system. The data obtained were processed using off-the-shelf commercial software. Details of these three elements form the subject of the following subsections.

\subsection{Water Tank and Propeller Rig}

This element of the experimental setup consisted of a water tank with a propeller rig mounted to it. The open-topped water tank was constructed from 8 - $\mathrm{mm}$ thick clear glass measured $600 \times 600 \times 600 \mathrm{~mm}^{3}$ (see Figure 2). Water was filled to a depth of $562 \mathrm{~mm}$ to allow for the propeller rig. The tank was placed on a rigid table that provided a stable platform for measurements.

Seeding for the PIV measurements was provided by SP Micro Balloons (@ RIPMax plc) because of their near-neutral buoyancy and suitable size for PIV measurements (average particle size $28 \mu \mathrm{m}$ ). The quantity of seeding was regulated by monitoring the output from the PIV system. The tank walls were mopped regularly to improve optical access that was otherwise impeded by leftover seeding on the tank walls. More seeding particles were added to compensate for those lost to cleaning. During the course of the experiments presented here, the water in the tank was replaced once.

The propeller rig was mounted on top of the water tank (see Figure 2). This consisted of a platform that fitted tightly over the top of the water tank and was mounted flush with one of the tank's inner walls, covering almost half of the exposed area.

The propeller (taken here to mean the wing/blade and the hub) was attached by means of a $15 \mathrm{~mm}$-diameter shaft to a second screw-driven mobile platform that was mounted on parallel rails running across the middle of the main platform. The horizontal position of the propeller shaft in the water tank was controlled manually by adjusting the screw on the mobile platform. This position was controlled to within $0.5 \mathrm{~mm}$. The vertical position of the propeller was fixed by the length of the shaft which placed it in the centre of the water tank. This arrangement enabled data for different spanwise and chordwise positions to be acquired without having to move the laser light sheet.

The propeller had only one blade — an aspect ratio 4 rectangular wing with $40 \mathrm{~mm}$ chord and $100 \mathrm{~mm}$ length — made from $2 \mathrm{~mm}$ thick steel plate for rigidity (see wing detail in Figure 2). The wing was embedded in the propeller shaft at $45^{\circ}$ angle-of-attack and such that rotation was about the midchord position at the wing root. The propeller shaft extended $7.5 \mathrm{~mm}$ into the wing, thus occupying only a little over $2 \%$ of the wing area (Figure 2).

The propeller was driven by a Nema17, 200-step-per-revolution hybrid stepping motor with a $0.36 \mathrm{Nm}$ holding torque. It was driven by a $8 \times$ microstepping bipolar drive operating at $20 \mathrm{~V}$ providing 1600 phase angles per revolution for smooth operation. Positioning accuracy was 400 steps per revolution $\left(0.9^{\circ}\right.$, half of a full step). By making optical measurements, it was verified that no steps were 'lost' in any of the experiments. The relatively low torques experienced by the propeller shaft for the current experiments obviated the need for a gearbox and the 


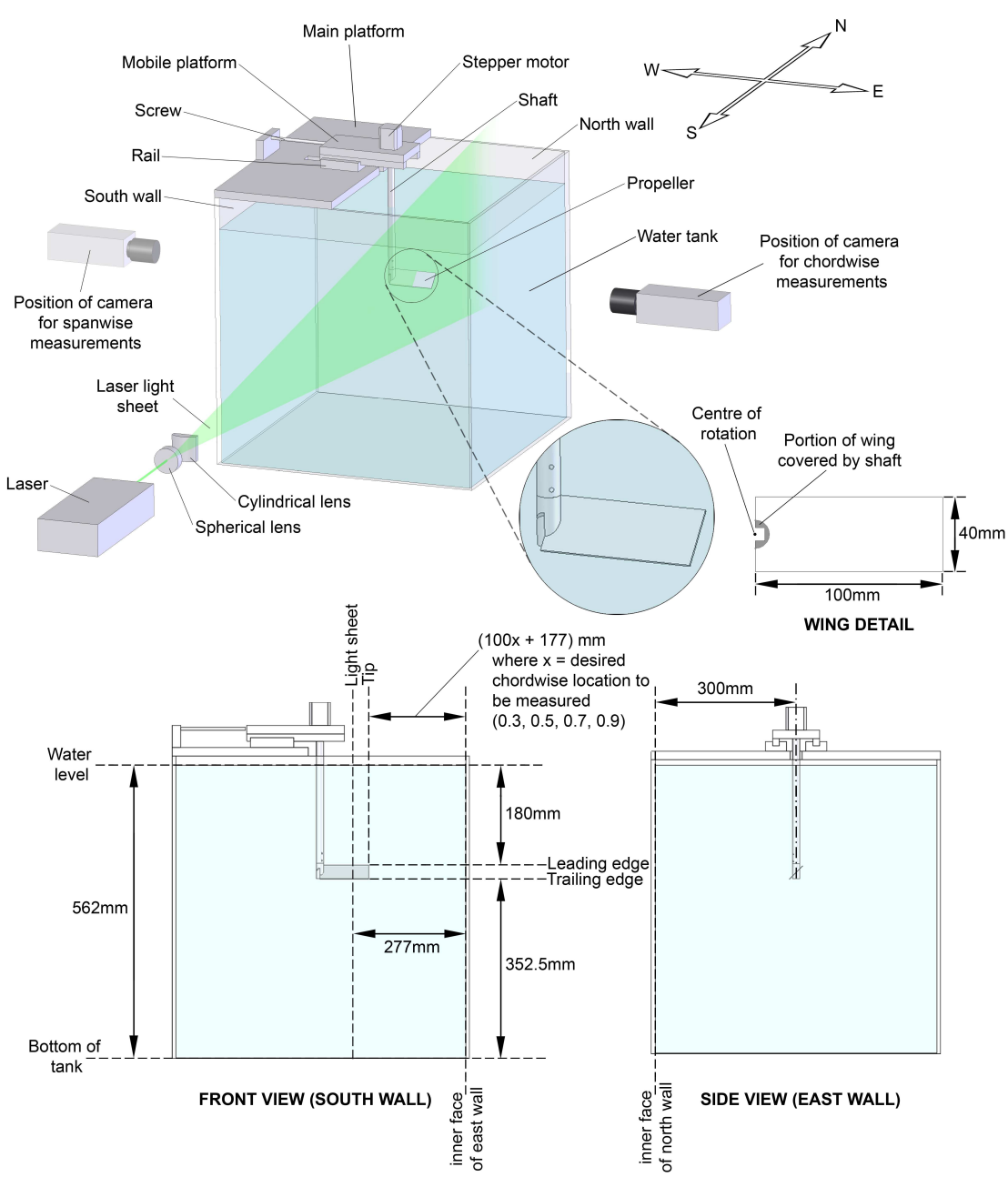

Fig. 2 Diagram of experimental setup with water tank, propeller rig, laser and camera; isometric view (top), front view (bottom left), side view (bottom right), wing detail (centre right)

propeller shaft was mounted directly onto the shaft of the motor. An opto-switch, which was mounted to the motor and triggered by a slotted disk on the motor shaft, was used for 'homing' to the reference datum and controlling the azimuthal position of the propeller.

The stepper motor was controlled by a Parallax Inc. protoboard, including a P8X32A chip (32-bit, $80 \mathrm{MHz}, 8$-core microcontroller) that was programmed with the required motions. Hardware counters and multiple cores made it possible to allow one core to generate step pulses while being monitored by a second core for low-latency PIV triggering. The motions were programmed via a PropStick USB-to-serial adapter using the PropTool IDE. 
Table 1 PIV acquisition parameters

\begin{tabular}{lcccc}
\hline & \multicolumn{2}{c}{ Spanwise Measurements } & \multicolumn{2}{c}{ Chordwise Measurements } \\
& $R e=500$ & $R e=15000$ & $R e=500$ & $R e=15000$ \\
\hline Area of interest $\left[\mathrm{mm}^{2}\right]$ & $70.5 \times 71$ & $70.5 \times 71$ & $109.6 \times 110.4$ & $109.6 \times 110.4$ \\
Image size $\left[\mathrm{px}^{2}\right]$ & $1008 \times 1016$ & $1008 \times 1016$ & $1008 \times 1016$ & $1008 \times 1016$ \\
Magnification & 0.129 & 0.129 & 0.083 & 0.083 \\
Lens aperture (f\#) & 2.8 & 2.8 & 2.8 & 2.8 \\
Calibration [px/mm] & 14.3 & 14.3 & 9.2 & 9.2 \\
Pulse separation [ms] & 30 & 1.2 & 70 & 1.2 \\
Observed maximum in- & $3-7.2$ & $5.1-10.6$ & $5.2-9.6$ & $3.7-7$ \\
plane displacement [px] & & & & \\
\hline
\end{tabular}

\subsection{PIV System}

The PIV system used in the experiment employed a Nd:YAG laser with a wavelength of $532 \mathrm{~nm}$ and a pulse width of $10 \mathrm{~ns}$. A set of lenses, consisting of a spherical lens with a negative focal length followed by a cylindrical lens with a positive focal length, was used to transform the beam into a light sheet. The light sheet was oriented such that it was parallel to and $277 \mathrm{~mm}$ away from the east wall of the water tank (see Figure 2). Image pairs were captured using a Kodak Mega Plus ES1.0 camera which had a CCD chip measuring $9.07 \mathrm{~mm}$ wide by $9.14 \mathrm{~mm}$ tall. The CCD chip size in pixels was $1008 \times 1016$, where each pixel was $9 \times 9 \mu \mathrm{m}^{2}$. The lens used on the camera was a $50 \mathrm{~mm}$ focal length Nikkor AFD set to a lens aperture (f\#) of 2.8. Lastly, the working distances (distance from camera to light sheet) used for the spanwise (along the wing span at constant chord positions) and chordwise (along the wing chord at constant span positions) measurements were $360.5 \mathrm{~mm}$ and $560.2 \mathrm{~mm}$, respectively.

\subsection{Data Processing}

Acquisition parameters used for both spanwise and chordwise measurements are summarised in Table 1. Listed are the areas imaged, the image sizes, magnification, lens aperture (f\#), calibration, pulse separation, and the observed range of maximum in-plane displacements using the indicated pulse separations. The acquired image pairs were processed using DaVis FlowMaster software version 6.2.3 by LaVision. The correlation function used was a standard cyclic fast Fourier transform-based algorithm which uses the equation

$$
C(\mathrm{~d} x, \mathrm{~d} y)=\sum_{x=0, y=0}^{x<n, y<n} I_{1}(x, y) I_{2}(x+\mathrm{d} x, y+\mathrm{d} y), \quad-\frac{n}{2}<\mathrm{d} x, \mathrm{~d} y<\frac{n}{2}
$$

where $I_{1}$ and $I_{2}$ are the image intensities from the first and second recording, respectively, $n$ is the interrogation window size, and $\mathrm{d} x$ and $\mathrm{d} y$ are the particle image displacements in the $x$ and $y$ directions, respectively. Details on this correlation 
function may be found elsewhere (Ronneberger et al, 1998). Second-order correlation, as described in Hart (1998), was also utilised. This multiplies the correlations of two interrogation windows overlapping by $50 \%$. It increases the signal-to-noise ratio and thus decreases correlation errors as the product of the correlation peaks is much greater in proportion to the products of the relatively smaller noise peaks. In addition, a Gaussian peak fit was utilised by the software to locate correlation peaks to within sub-pixel resolution.

Using the described correlation function with second-order correlation, an adaptive multiple pass interrogation algorithm was employed using a coarse 'initial' grid progressing to a fine 'final' grid. In this method, a 'reference' vector field is computed in the first pass using an initial interrogation window size. The reference vector field is then continually refined in subsequent passes using a progressively finer grid. In the final pass the refined reference vector field is used to offset the final interrogation windows. This increases the number of matched particles between two images and thus optimises the detectability of the correlation peak and minimises measurement error (Keane and Adrian, 1992). The error occurs since fewer particles leave the final interrogation window as a consequence of the fact that the final interrogation window displaces with the particles. This method also allows final interrogation windows smaller than the particle image displacements to be used (Raffel et al, 1998). Deformed interrogation windows were also employed which further increases the number of matched particles and the signal-to-noise ratio. A total of four passes were utilised starting with an initial interrogation window size of $32 \times 32 \mathrm{px}^{2}$ in the first pass progressing to $16 \times 16 \mathrm{px}^{2}$ and $12 \times 12 \mathrm{px}^{2}$ in the subsequent two passes, followed by a final interrogation window size of $8 \times 8 \mathrm{px}^{2}$ in the final pass with a $0 \%$ overlap.

Between passes from the initial to final interrogation window size, the median filter proposed by Westerweel (1994) was utilised to locate spurious vectors and replace them by interpolation. It is important to catch spurious vectors between passes so that the final interrogation window will not be offset by the wrong value. In this filter, a 'median' vector is computed from the median $u$ and $v$ components of the eight neighbours of the vector in question. The vector in question is then rejected and interpolated if the absolute difference between it and the median vector is above some threshold. The threshold used was a specified multiple of an rms vector calculated from the rms of the $u$ and $v$ components of the eight neighbours of the vector in question. Problems with this type of filter typically occur around empty areas in the vector field where there are fewer neighbours for comparison (Raffel et al, 1998). A problem of this nature was encountered at the wing leading edge where too stringent a threshold was found to alter flow structures incorrectly (Figure 3). It was found that a threshold of $4.75 \times(\mathrm{rms}$ of neighbours) was suitable, as it identified spurious vectors but did not alter flow structures that were observed without the median filter.

The number of vectors in a given vector field which had to be inserted via interpolation either due to loss of correlation in the final interrogation window from insufficient seeding or due to removal of spurious vectors was on average $\sim 2.5 \%$ with a minimum of $0.5 \%$ and maximum of $7 \%$. This was based upon a sample size of 40 vector maps consisting of single measurements from azimuth positions $5^{\circ}, 30^{\circ}, 90^{\circ}$ and $270^{\circ}$ for all spanwise and chordwise locations for both Reynolds numbers. The border of interpolated vectors framing each vector field, 

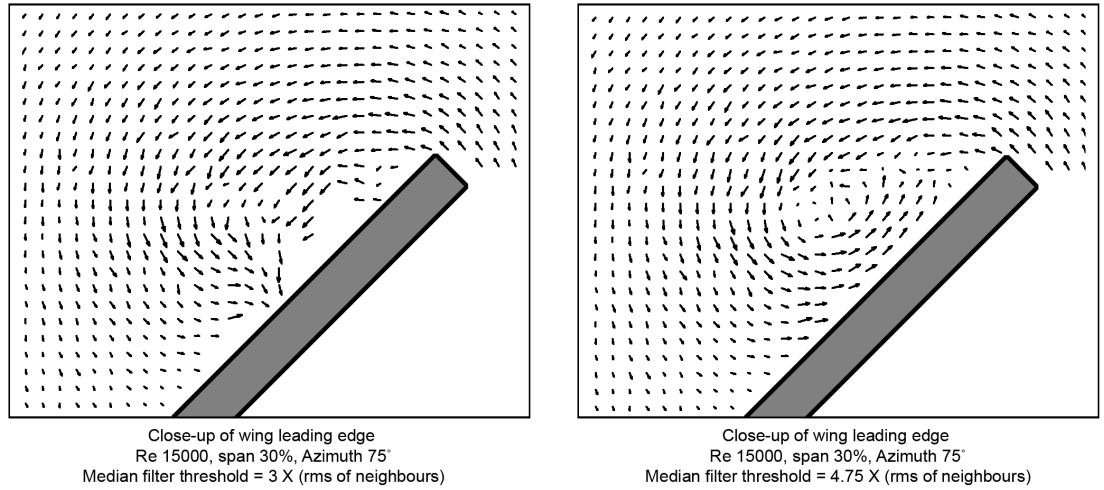

Fig. 3 Effect of median filter thresholding shown for too stringent (left) and adequate (right) thresholds

which occur due to loss of correlation at the edges of the image and which are present regardless of interrogation window size or seeding density, was ignored in this calculation. In general, it was observed that greater azimuth positions (see Figure 5 for definition of azimuth) resulted in a greater number of interpolated vectors. The observed level of interpolated vectors was mainly a consequence of the small final interrogation window size. However, it was felt that this level of interpolated vectors was acceptable since vortex structures, such as the leadingedge vortex, were of primary interest in the present experiment, and small interrogation window sizes are better for resolving such structures. This is because vortex structures contain large velocity gradients within small areas, which results in a biasing effect towards lower velocities for larger interrogation window sizes. By using smaller interrogation window sizes, this decreases the velocity gradients within the interrogation window, which in turn decreases biasing error (Willert and Gharib, 1991). In addition, Willert and Gharib also note that the velocity gradients and the rotational motions near the core of a vortex cause a distortion or a loss of the correlation peak, in which case a small interrogation window size should be used.

\subsection{Experimental Procedure}

The various components of the experimental setup were arranged as shown in Figure 4 . The experiments were carried out in a cool room $\left(15 \pm 5^{\circ} \mathrm{C}\right)$. Both chordwise and spanwise flow measurements were made at various wing azimuthal positions. The wing was started from rest and moved through an azimuth angle $\psi$ at constant speed to reach the test position. PIV measurements were made as the wing passed through $\psi$. The wing continued moving to $\psi+5^{\circ}$, then stopped and backed up to the next starting position. Settling time (see Section 4.3) was allowed before the next sweep from the new starting position commenced.

The test conditions are summarised in Table 2. Two wing speeds, giving $R e=$ 500 and $R e=15000$, were used to capture flow properties relevant to insects and micro air vehicles, respectively. Reynolds number is defined with respect to mean 


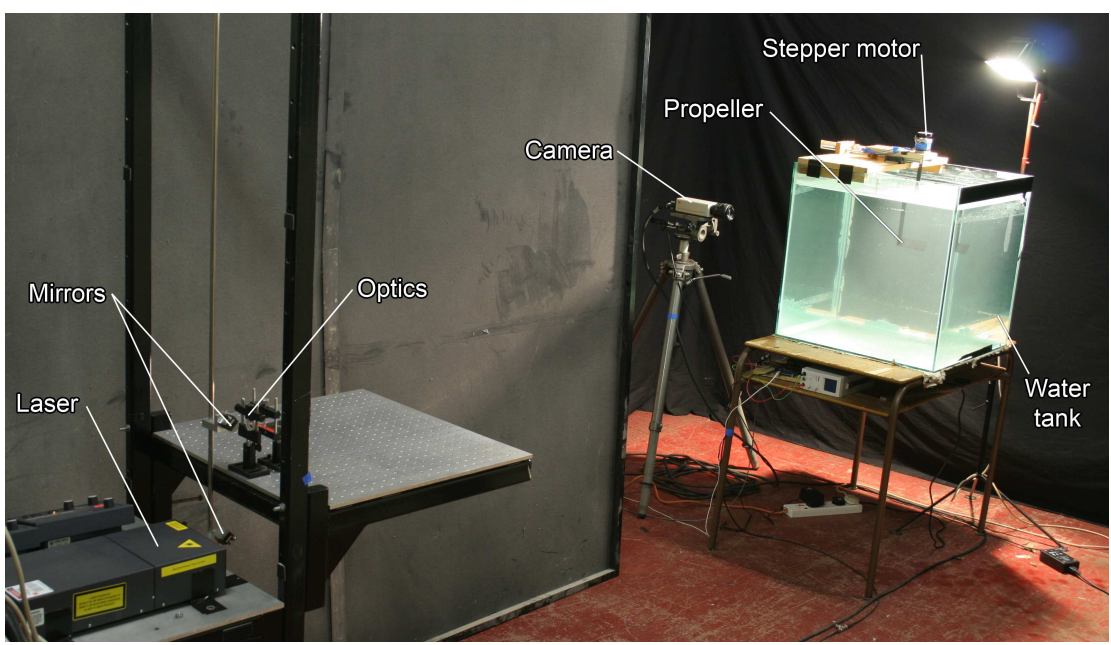

Fig. 4 Experimental setup photo

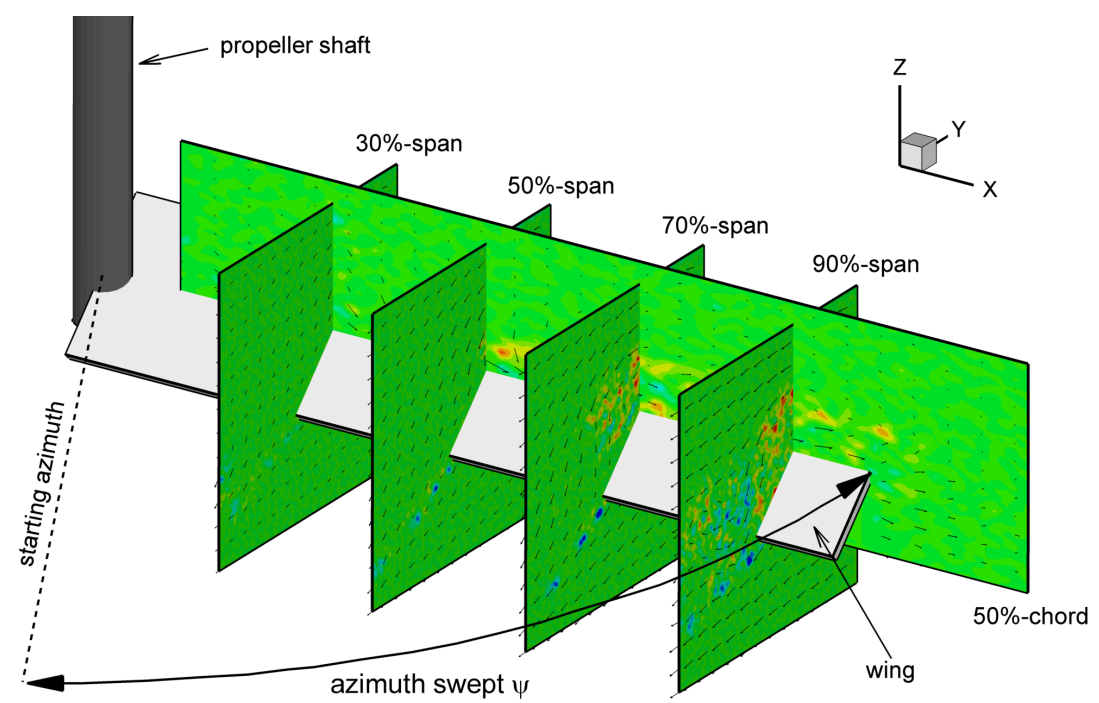

Fig. 5 Diagram showing arrangement of planes captured in the PIV experiments with the wing shown at the trigger position

wing chord $(40 \mathrm{~mm})$ and mean wingtip speed $(100 \mathrm{~mm} \times 2 \pi f)$, where $f$ is rotation rate $(0.126 \mathrm{~Hz}$ and $3.77 \mathrm{~Hz}$ for $R e=500$ and $R e=15000$, respectively, in water, density $\rho=998 \mathrm{~kg} / \mathrm{m}^{3}$ and viscosity $\left.\mu=1.003 \times 10^{-3} \mathrm{~Pa} \cdot \mathrm{s}\right)$. Due to the settling times required between tests (see Section 4.3) and in the interest of capturing the same positions for both flow speeds (to enable like-for-like comparisons), the flow was measured at 12 azimuthal positions. Except for the spanwise positions 
for $R e=500$, all experiments were run twice and the results shown here are for the flow fields obtained by averaging the two datasets.

Table 2 Test cases

\begin{tabular}{ll}
\hline Spanwise Measurement Planes (\% span) & $30,50,70,90$ \\
Chordwise Measurement Planes (\% chord) & 50 \\
Azimuthal Positions (deg) & $5,10,15,30,45,60,75$, \\
& $90,135,180,225,270$ \\
Reynolds Numbers & 500,15000 \\
\hline
\end{tabular}

Defining the walls of the water tank such that their outward normals are compass directions, then the light sheet from the laser entered the tank from the South wall and left by the North wall (see Figure 2). For the chordwise ( $Y-Z$ planes; see Figure 5) flow measurements, the camera was placed facing the East wall and looking into the tank. The propeller rotated counterclockwise (as seen from above) so it was not possible to photograph the spanwise ( $X-Z$ plane; see Figure 5) measurements from the same location (in order to maintain the orientation of the wing's angle of attack), and the camera had to be repositioned facing the West wall and pointing into the tank (Figure 2).

The squareness of the water tank was measured with a set square and found to be correct. The laser light sheet was then aligned parallel to the East face by ensuring that it crossed the North and South walls at the same distances from the East wall. The position of the propeller wing was calibrated by aligning it square with the East wall. The camera was aligned differently for the chordwise and spanwise flow measurements.

For the chordwise measurements, the laser and camera of the PIV system were triggered when the wing's spanwise axis was parallel to the North wall and pointed towards the East wall into the camera. The camera was aligned such that the wing section was edge-on in the camera's view. For the spanwise measurements, the camera and laser were set to trigger when the wing's spanwise axis was parallel to the East wall and was pointing towards the South wall and in the direction of the laser source. The camera was aligned by ensuring the leading and trailing edges of the wing were horizontal to within a pixel width, and that the wing tip was similarly vertical to within a pixel width.

The positions of the chordwise sections ( $Y-Z$ planes) were measured by reading off where the laser light sheet 'cut' the wing. For the positions of the spanwise sections ( $X-Z$ plane), the distance of the leading edge of the wing from the East wall was used. This was referenced to the position of the laser light sheet from the East wall.

To reduce errors, the laser, camera and water tank were kept stationary. Measurements at the various spanwise, chordwise and azimuthal positions of the wing were facilitated by moving the propeller alone. For example, for an azimuthal position of $90^{\circ}$, the wing was started $90^{\circ}$ before the trigger position. Similarly, for various spanwise measurement positions, the drive shaft of the propeller was po- 
sitioned by adjusting the screw-driven platform to which the propeller drive shaft was attached. The drive shaft was started impulsively in all cases.

A typical experimental run started with stirring the tank to distribute seeding and to allow particles that had risen to the surface to descend into the tank. Ample time was allowed to let the flow settle before moving the wing and taking measurements (see Section 4.3 below). The experimental programme went through all azimuthal positions, firing the laser and triggering the camera at the correct instances. For the case of $R e=15000$, a typical run lasted about 3 min while for $R e=500$ it was of the order of $4 \mathrm{hrs}$.

\section{Uncertainty Analysis}

\subsection{Seeding Response}

Associated with seeding is an error arising from the degree to which the particles truly follow the flow. Particles with small diameters and densities similar to the fluid are very good at following the flow. However, small particle diameters are more difficult to image as they scatter less light; therefore, a balance must be struck between particle diameter and particle image size. As noted earlier, the micro glass balloons used in the current experiments as the seeding were sized to be on average about $28 \mu \mathrm{m}$. This is very similar to the seeding particles used in an experiment by Graham and Soria (1994), who used hollow glass spheres with a diameter $<30 \mu \mathrm{m}$ which showed to have a good tracking capability in water. Based upon the laser wavelength, particle size, magnification and lens aperture (f\#) used for the present experiment, the particle image size was estimated to be on average $0.6 \mathrm{px}$ and $0.5 \mathrm{px}$ for the chordwise ( $Y-Z$ planes) and spanwise $(X-$ $Z$ plane) measurements, respectively. However, in the captured images the actual size was observed to be on average $\sim 1.8 \mathrm{px}$ and $\sim 1.6 \mathrm{px}$, respectively. This discrepancy could be attributed to seeding particles clumping together, or to a lens that was slightly out of focus, which is a method used in practice for increasing the particle image size (Raffel et al, 1998). In either case, the seeding response was evaluated by observing the gravitationally induced flow velocity originating from the difference in density between the seeding particles and the fluid. A 'settling time' experiment, which will be discussed in detail in Section 4.3 below, was performed to observe the motion of the fluid at various time intervals following a wing translation. As will be shown later, when the fluid had settled, the mean vertical velocity was observed to be essentially zero, which suggests that the difference in density between the seeding particles and the fluid was minimal. This, in conjunction with the fact that the velocity lag of a particle in an accelerating fluid is proportional to the difference in density between the particle and the fluid according to the Stokes drag law (Raffel et al, 1998), led to the assumption that the seeding response error was negligible.

\subsection{PIV Error}

Errors in PIV measurements can be divided into three forms: outliers, mean-bias errors, and root mean square (rms) errors (Huang et al, 1997). Outliers which are 
spurious vectors arising from particle mismatches, were identified and removed using the median filter discussed in Section 3.3. The other two forms of error will now be discussed and evaluated.

RMS error is simply the deviation of a measured particle image displacement from the mean displacement, and is given by Huang et al (1997) as

$$
\sigma=\sqrt{\frac{\sum_{i=1}^{N}\left(d_{i}-d_{m}\right)^{2}}{N}}
$$

where $d_{i}$ is the measured particle image displacement, $d_{m}$ is the mean of the measured particle image displacements, and $N$ is the number of displacements. There are many factors that affect rms error, such as particle image size and density. These are discussed in detail in Huang et al (1997) and Raffel et al (1998). To quantify rms error for the present experiment, the approach used was that of Willert and Gharib (1991), in which rms error is measured by processing particle image pairs where the particles have been displaced by an amount that is known reliably. Using this approach, the water tank was left undisturbed for one hour and image pairs were captured using a pulse separation of $1 \mu \mathrm{s}$. This short pulse separation in conjunction with an undisturbed tank meant that the actual displacement of the particle images between pulses was confidently known to be zero. Therefore, any displacement that was measured was random error. Five image pairs were taken for both magnifications listed in Table 1, and were processed using the same method described in Section 3.3. The above equation was then applied to each set of vector maps to calculate the rms error for each magnification. This revealed a rms errors of $0.43 \mathrm{px}$ and $0.35 \mathrm{px}$ for the magnifications used in the spanwise and chordwise measurements, respectively. It should be noted that for particle image displacements greater than zero the rms error increases. However, by offsetting the final interrogation window as the present experiment does, the particle image displacement relative to the final interrogation window is close to zero. Therefore, the prediction of the rms error in the present experiment based upon measuring a zero particle image displacement is sufficient.

Mean-bias error is the difference between the mean measured particle image displacement and the actual particle image displacement. A side effect of FFTbased cross-correlations is that they are biased towards lower velocities. This is because they introduce a weighting factor which decreases with increasing velocities; thus, lower velocities are favoured since they have higher weighting factors (Keane and Adrian, 1990). Details on this may be found in Raffel et al (1998), Keane and Adrian (1990) and Westerweel (1997). The size of this error is directly proportional to the particle image displacement and the velocity gradient within an interrogation window; thus, if these are minimised then mean-bias error may be made negligible (Keane and Adrian, 1990). From this, it can be argued that mean-bias error in the present experiment was negligible since velocity gradients and particle image displacements were minimised through use of a small final interrogation window size and an offset, respectively.

In conclusion, the total error in the present experiment is dominated by rms error as all other forms of error were found to be negligible. This is in agreement with the observation that for particle image sizes $\gg 1 \mathrm{px}$, rms error will dominate (Westerweel, 1997), where the particle image sizes observed were on 
average $\sim 1.8 \mathrm{px}$ and $\sim 1.6 \mathrm{px}$ for the two measurement cases. Thus, with an rms error of $0.43 \mathrm{px}$ for the chordwise measurements, this leads to a measurement uncertainty of $\pm 0.001 \mathrm{~m} / \mathrm{s}$ and $\pm 0.025 \mathrm{~m} / \mathrm{s}$ for the $R e=500$ and $R e=15000$ cases, respectively. Similarly, for the spanwise measurements, in the $R e=500$ and $R e=15000$ cases an rms error of 0.35 px leads to a measurement uncertainty of $5.4 \times 10^{-4} \mathrm{~m} / \mathrm{s}$ and $\pm 0.032 \mathrm{~m} / \mathrm{s}$, respectively.

\subsection{Other Considerations}

Since the wing was rotated while the measurement system was kept fixed, only one measurement could be made per pass. To make several measurements, the propeller was started from different starting azimuths. One of the most important considerations in the experiments, therefore, was the settling time between runs. To measure the 'stillness' of the flow in the tank, PIV measurements were made of the area of interest for various settling times after the wing motion ceased in preparation for a subsequent test. This was done for the largest azimuthal position $\left(\psi=270^{\circ}\right)$ and the $90 \%$-span position for which the flow disturbances were envisaged to be the greatest.

The results from this study are presented in Figures 6 and 7. All graphs are normalised with respect to the wing velocity at the 90\%-span position. Figure 6 shows polar plots for the normalised velocity field for $R e=500$. The position of the points in the plot give the magnitude of the velocity vectors and their direction in the $x-y$ plane. For a completely still flow, all points should lie in the centre of the plots. Based on these results, a settling time of 20 min was chosen for the wing PIV measurements. This corresponded to less than $10 \%$ normalised velocity anywhere in the area of interest and a mean normalised flow velocity of less than $2 \%$ (see also Figure 7(a)). As may be evident from Figure 7(a), there are diminishing returns for longer settling times, owing to dropping seeding levels and movement arising from the buoyancy and collision of seeding particles.

For $\operatorname{Re}=15000$, the settling time between runs was fixed at $10 \mathrm{~s}$ (see Figure 7(b)). For this interval, mean normalised flow speeds were less than $0.1 \%$.

There are other possible sources of discrepancies that are not easily quantifiable. Because wing position in the tank was altered for each of the chordwise measurements (30\%- to $90 \%$-span), the propeller was not always in the middle of the tank horizontally; it was off-centre by $7-67 \mathrm{~mm}$ which could give rise to errors due to wall effects and asymmetry. Given the size of the wing relative to the tank, however, these effects are assumed to be minimal. There are also possible errors resulting from the recirculating flow due to wall effects; minimum wall clearance varied from 5.2-6.7 mean wing chords. This effect was minimised by the short run durations and the settling times between runs.

\section{Results and Discussion}

The PIV image pairs were processed by the DaVis FlowMaster software to produce velocity vector fields. From these, the flow was visualised using vorticity 


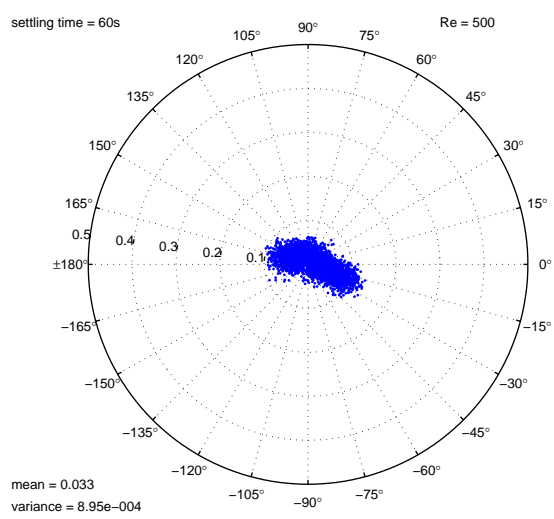

(a) $1 \mathrm{~min}$

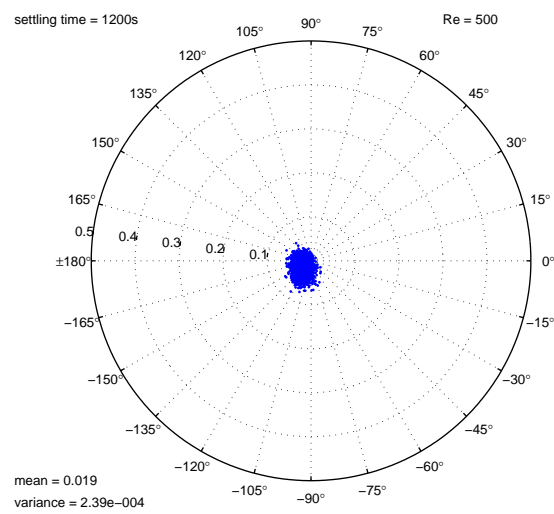

(c) 20 mins

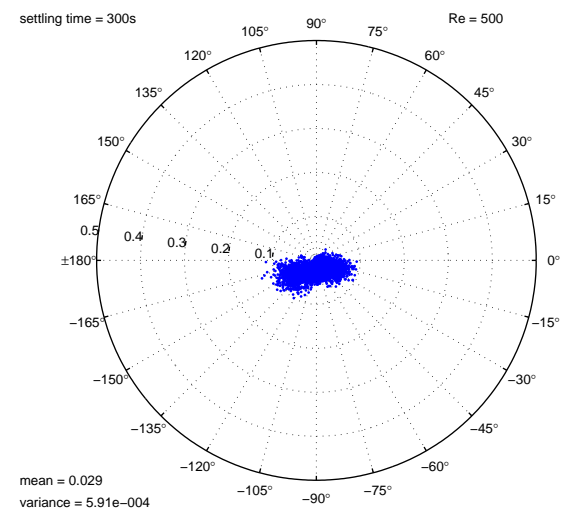

(b) 5 mins

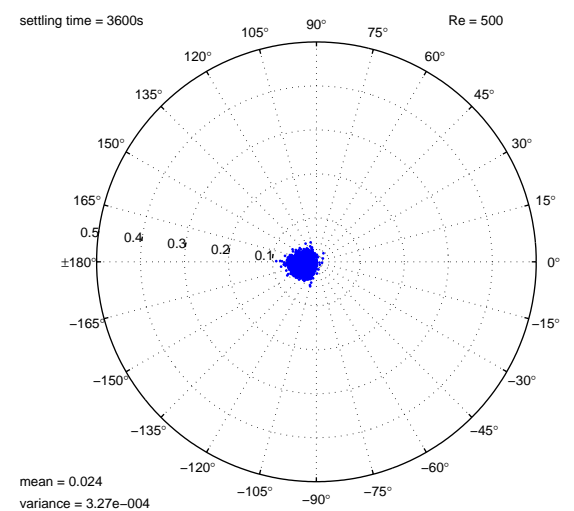

(d) $1 \mathrm{hr}$

Fig. 6 Plots showing dispersion of normalised velocity for various settling times for $\operatorname{Re}=500$ at $90 \%$ span (velocities normalised with respect to wing speed at $90 \%$ span)

contour plots overlayed with velocity vectors, and by applying line integral convolution (LIC) to the velocity vector fields (Knowles et al, 2006) to produce flow field images.

Data were generated according to the test cases described in Table 2. They were for 2 Reynolds numbers $(R e=500$ and $R e=15000)$ in 4 chordwise planes (at 30\%-, 50\%-, 70\%- and 90\%-span positions) and 1 spanwise plane (at 50\%chord position). These planes are shown in Figure 5.

A typical complete set of results is shown in Figure 8. The general trend from the results is as follows. As the propeller wing starts up, leading- and trailingedge vortices are formed $\left(\psi \approx 5^{\circ}\right)$. The vortex at the leading edge begins to grow while that formed at the trailing edge is shed soon after $\left(\psi \approx 10^{\circ}\right.$ or $\left.15^{\circ}\right)$. At startup, the flow field around the wing is more or less horizontal but as the wing sweeps larger angles, the flow has a definite downward component. This effect becomes less pronounced for outboard regions of the wing. As shown later (see 


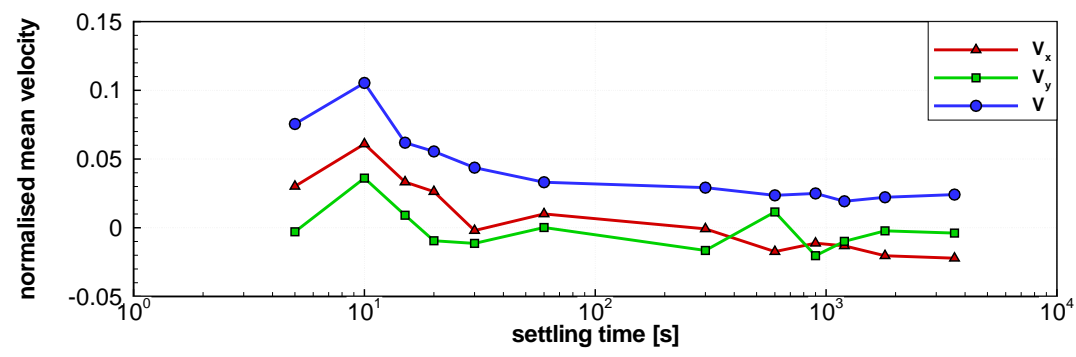

(a) $\operatorname{Re}=500$

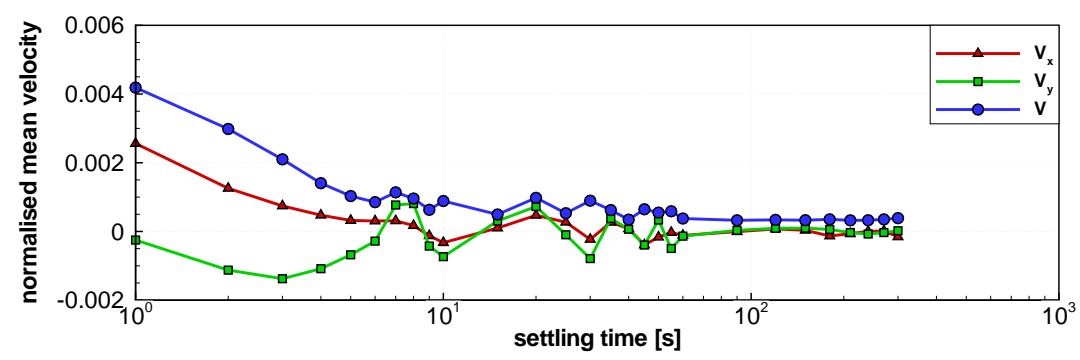

(b) $\operatorname{Re}=15000$

Fig. 7 Mean normalised velocities for $R e=500$ and $R e=15000$ at $90 \%$ span for various settling times ( $V_{x}$ is mean $x$-velocity, $V_{y}$ is mean $y$-velocity and $V$ is mean velocity magnitude)

Figures 17 and 18), immediately following startup the flow from the leading-edge vortex is predominantly chordwise, but as the wing continues to sweep, the flow vector becomes increasingly spanwise in the outboard direction. This indicates a spiralling leading-edge vortex structure that subsequently coalesces with the tip vortex, similar to that seen during the sweeping phase of the half-stroke on insectlike flapping wings (e.g. Ellington et al, 1996).

To consolidate the results and assist with analysing trends, the data were classified into effects of spanwise position (Section 5.1) and effects of Reynolds number (Section 5.2), and key results from these are presented here.

\subsection{Effect of Spanwise Position}

The effect of spanwise position on the chordwise flow field is shown in Figures 918. Three azimuthal positions have been chosen for these comparisons $\left(10^{\circ}, 90^{\circ}\right.$ and $270^{\circ}$ ) so that flow features relating to startup, mid-stroke and end of (a long) stroke may be studied. Figures 9-11 show the chordwise flow field using vorticity contours and LIC images, and Figure 15 contains line plots of the horizontal velocity profile through a vertical section at the midchord point of the wing, all for $\operatorname{Re}=500$. Figure 17 shows the spanwise flow field for the same case. For $\operatorname{Re}=15000$, the corresponding plots are shown in Figures 12-14, Figure 16 and Figure 18. 

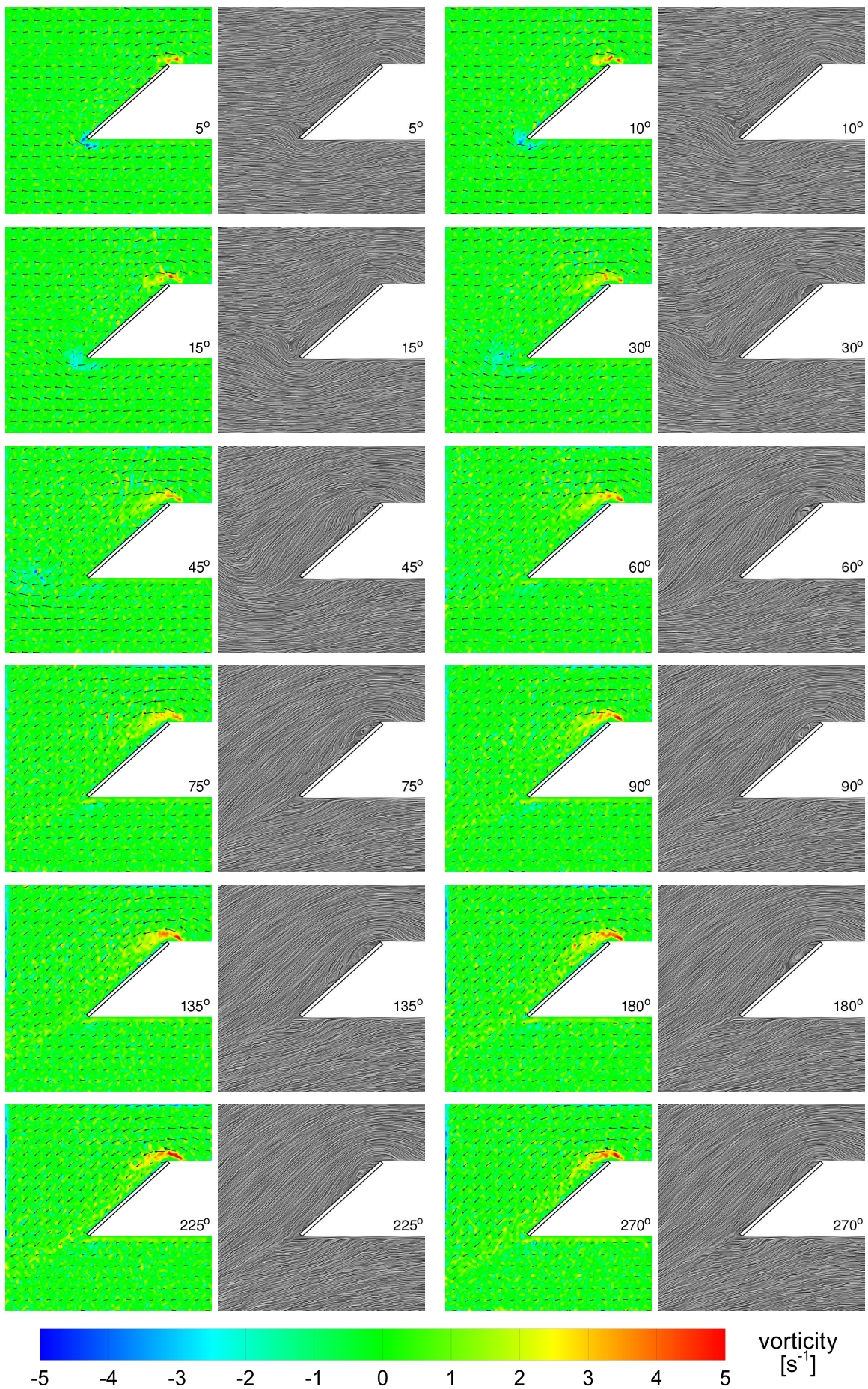

vorticity
$\left[\mathrm{s}^{-1}\right]$

Fig. 8 Results for $R e=500,30 \%$-span position and azimuths as indicated, shown by plots of contours of vorticity overlayed with velocity vectors (columns 1 and 3) and LIC images (columns 2 and 4 ) 

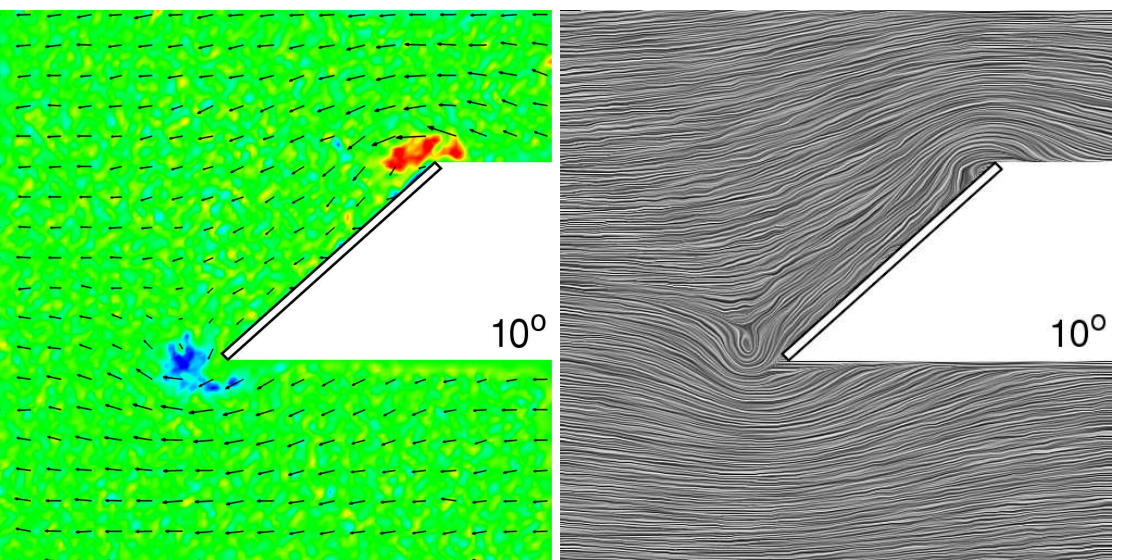

(a) $50 \%$-span

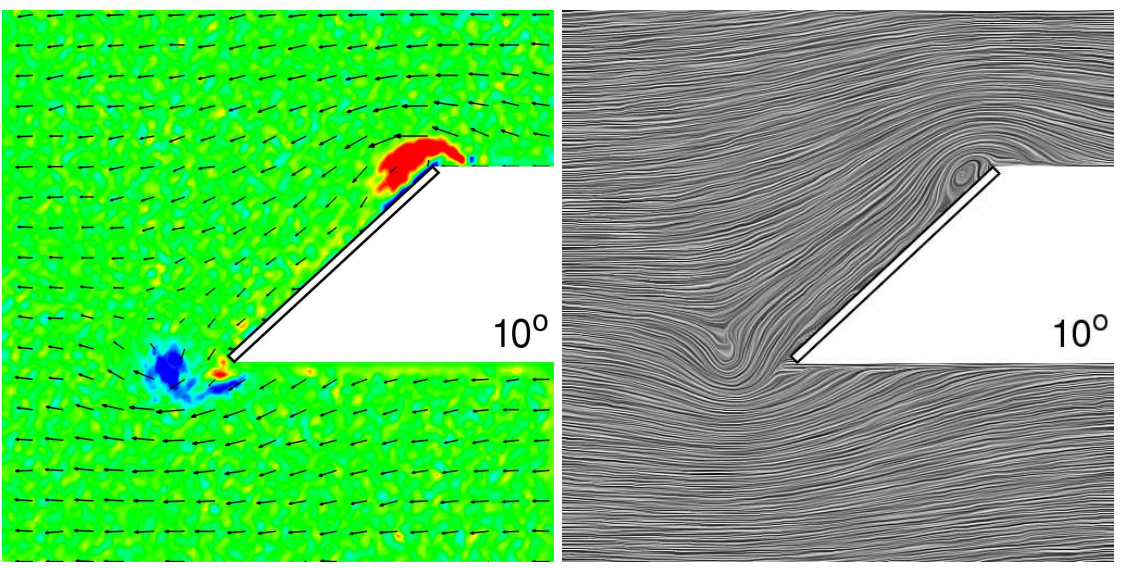

(b) $70 \%$-span
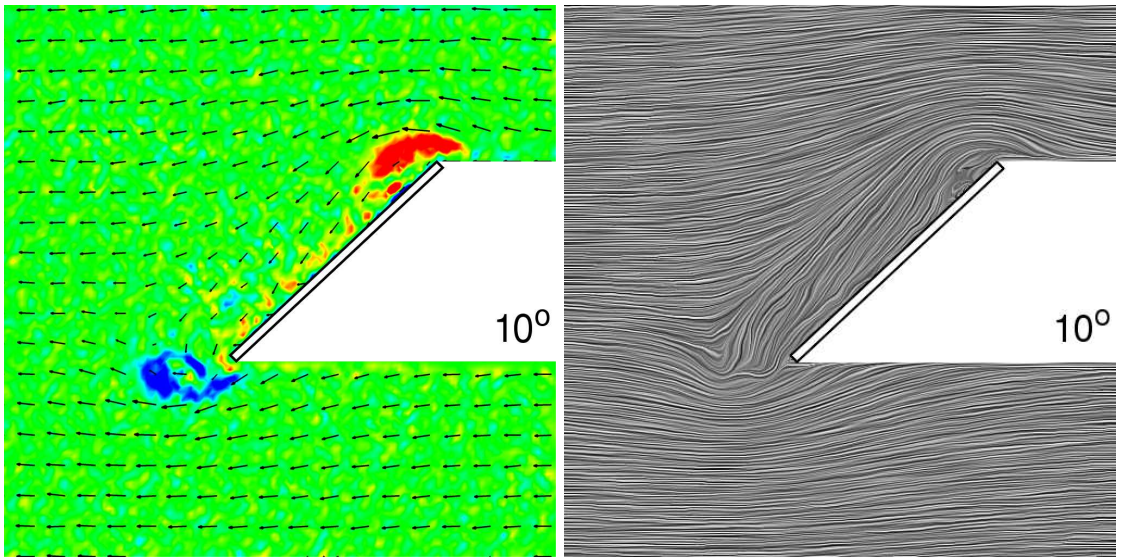

(c) $90 \%$-span

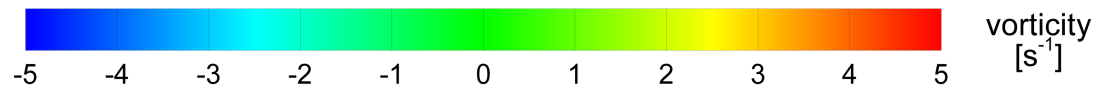

Fig. 9 Effect of spanwise position on chordwise flow for $R e=500$ at $10^{\circ}$ azimuth, shown by plots of contours of vorticity overlayed with velocity vectors (left) and LIC images (right) 


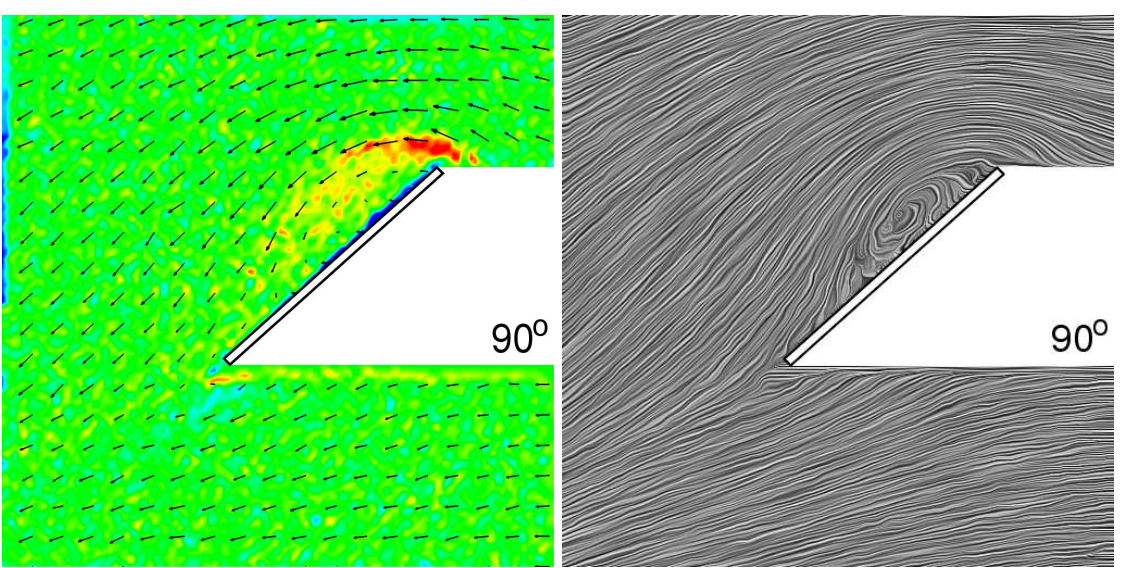

(a) $50 \%$-span
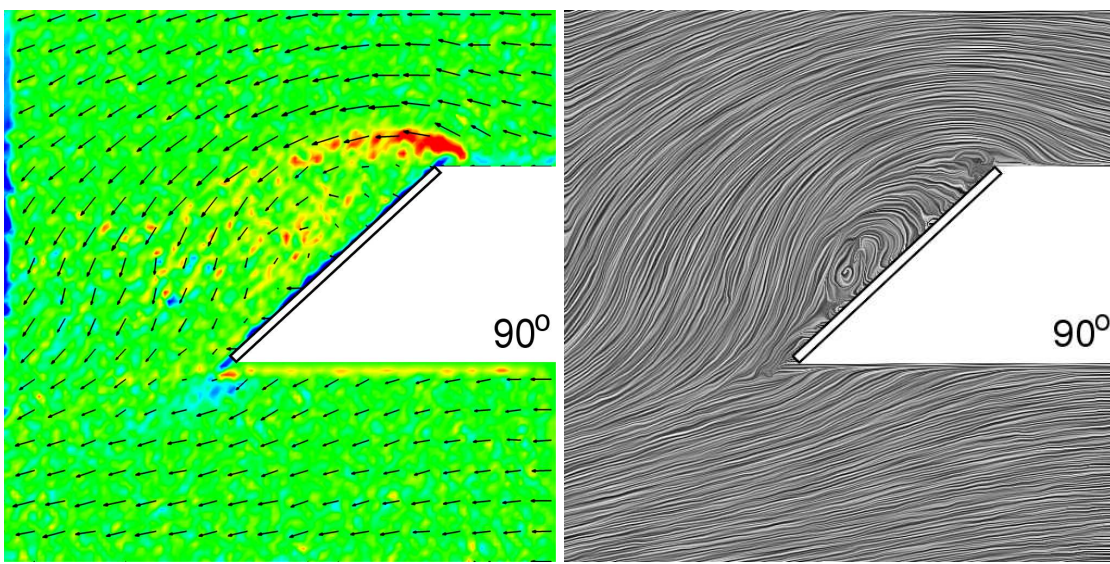

(b) $70 \%$-span
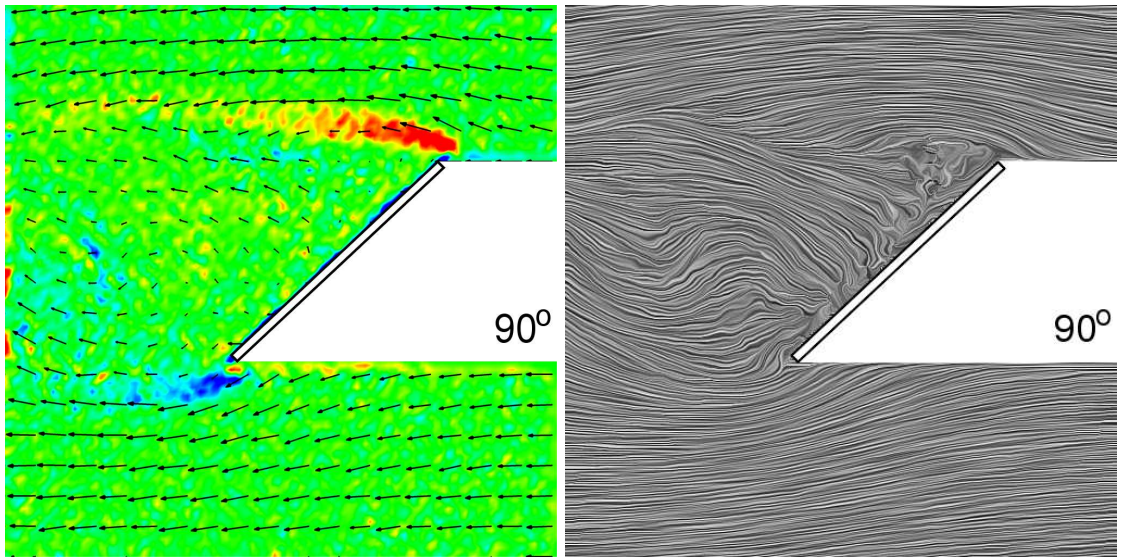

(c) $90 \%$-span

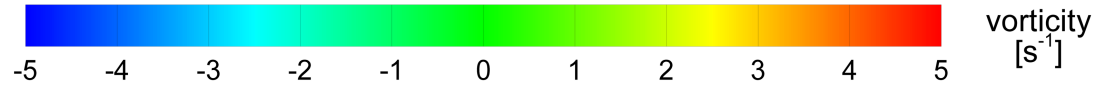

Fig. 10 Effect of spanwise position on chordwise flow for $R e=500$ at $90^{\circ}$ azimuth, shown by plots of contours of vorticity overlayed with velocity vectors (left) and LIC images (right) 

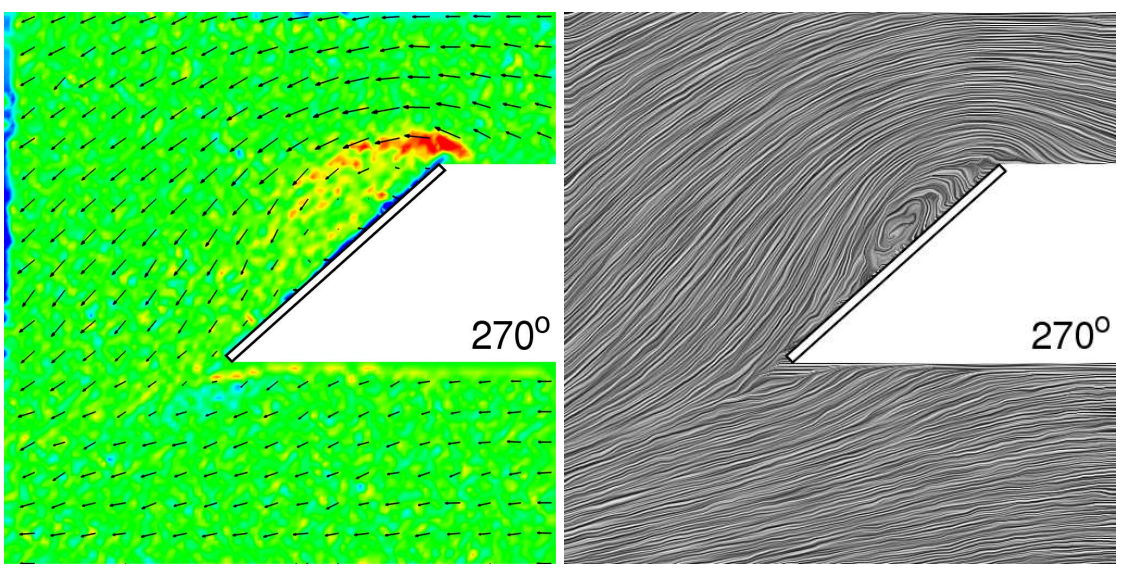

(a) $50 \%$-span
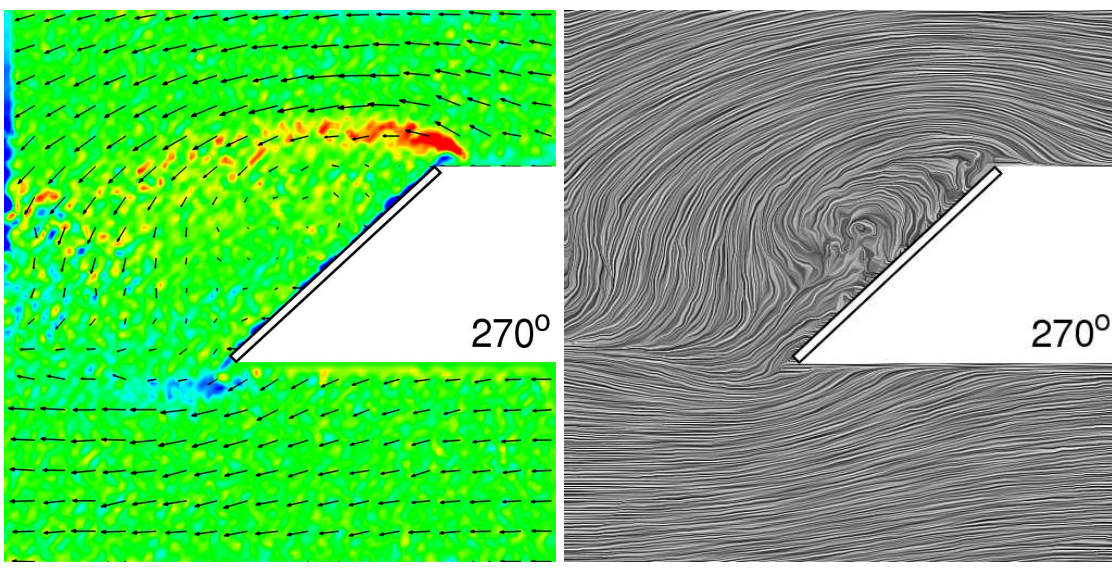

(b) $70 \%$-span
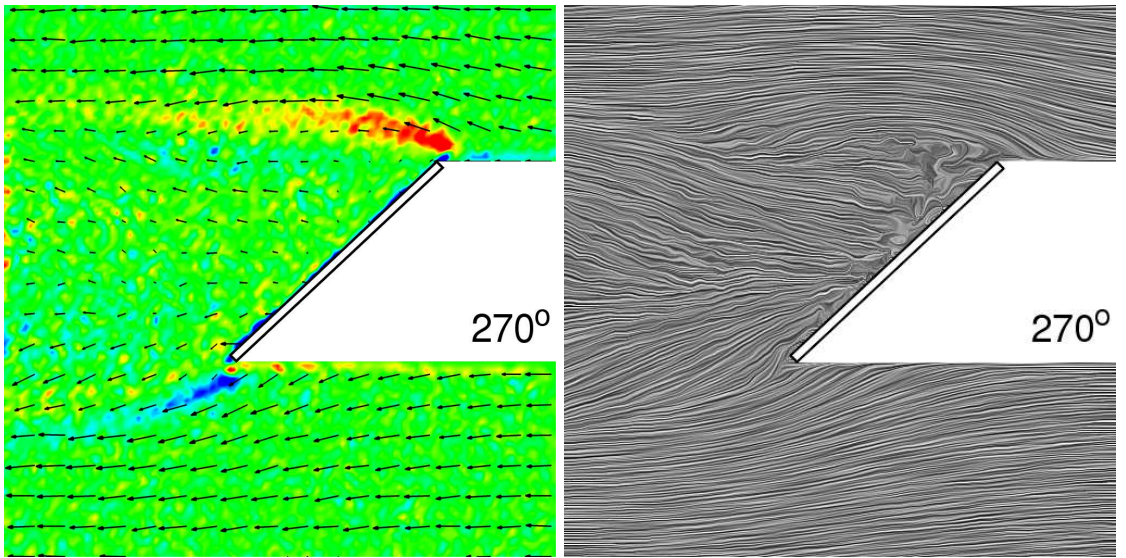

(c) $90 \%$-span

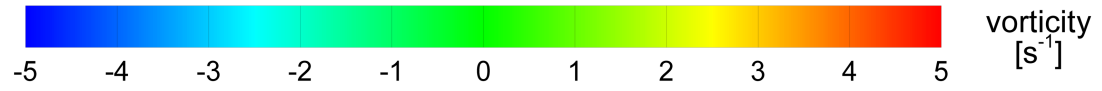

Fig. 11 Effect of spanwise position on chordwise flow for $R e=500$ at $270^{\circ}$ azimuth, shown by plots of contours of vorticity overlayed with velocity vectors (left) and LIC images (right) 


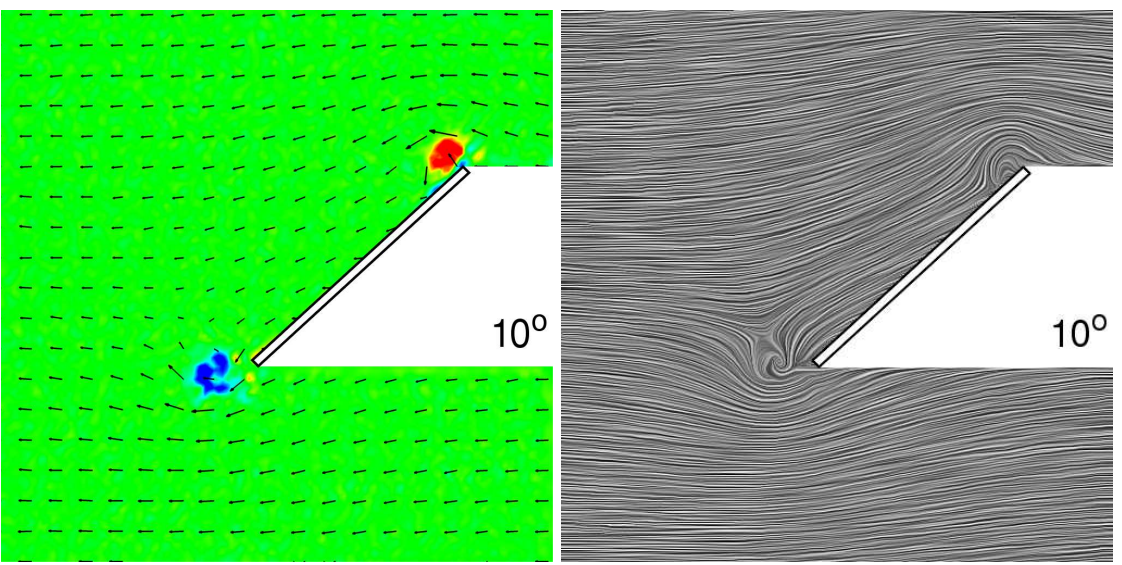

(a) $50 \%$-span

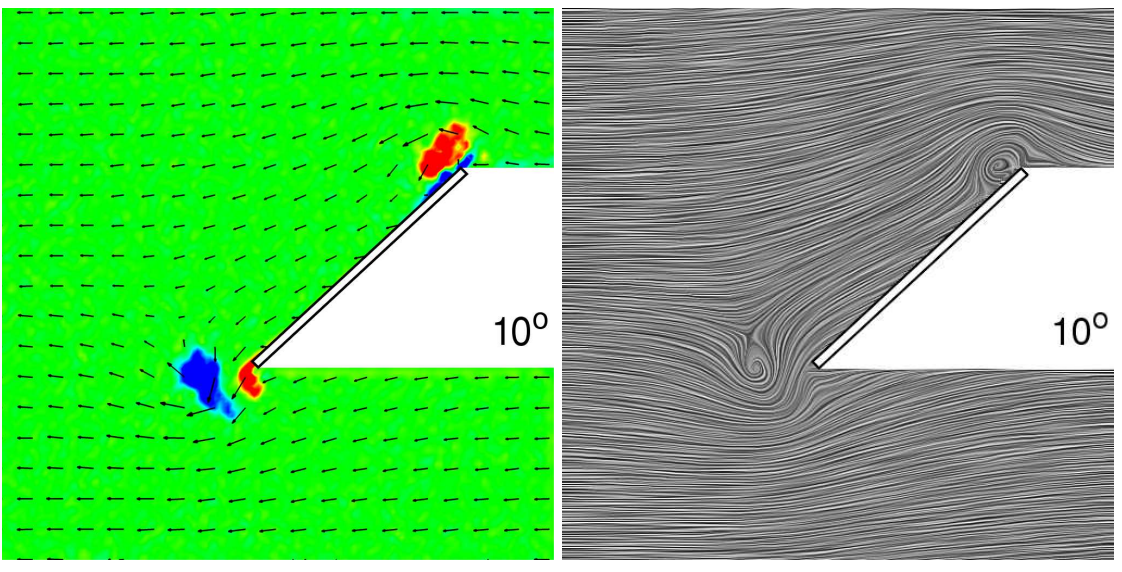

(b) $70 \%$-span

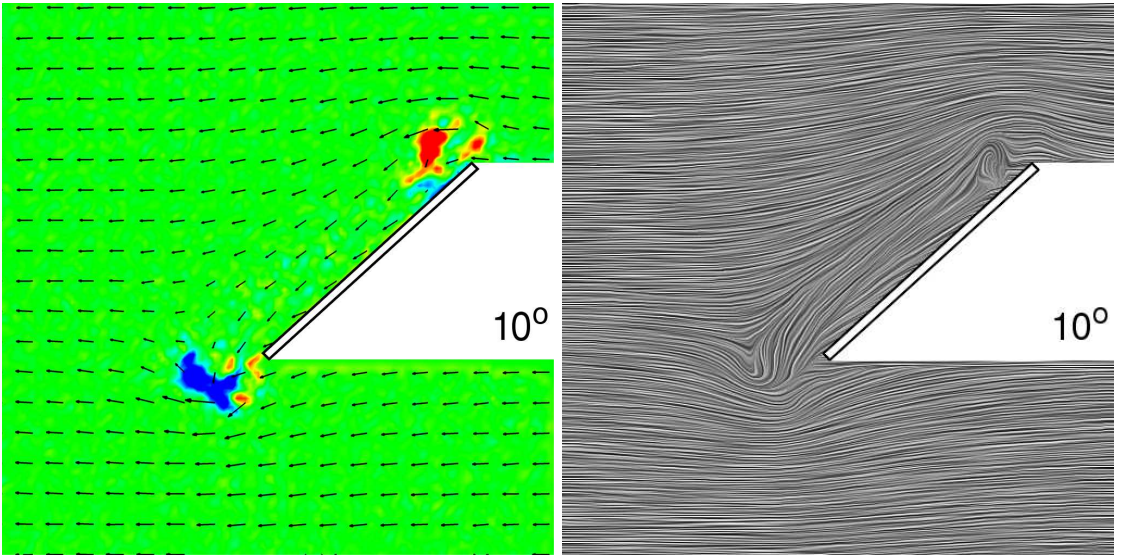

(c) $90 \%$-span

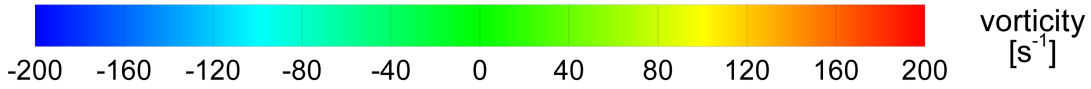

Fig. 12 Effect of spanwise position on chordwise flow for $R e=15000$ at $10^{\circ}$ azimuth, shown by plots of contours of vorticity overlayed with velocity vectors (left) and LIC images (right) 

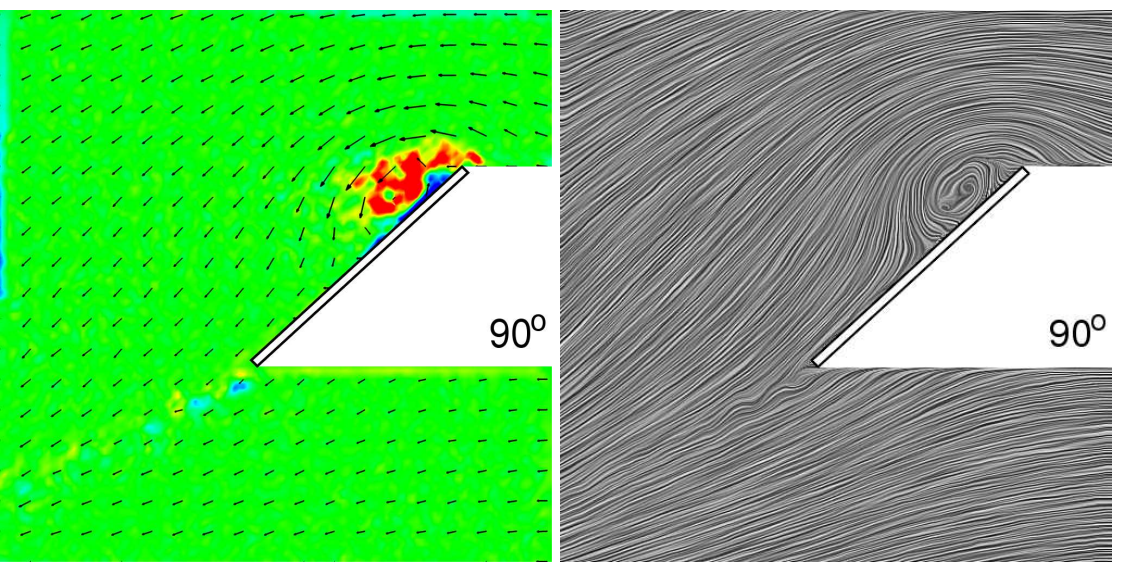

(a) $50 \%$-span
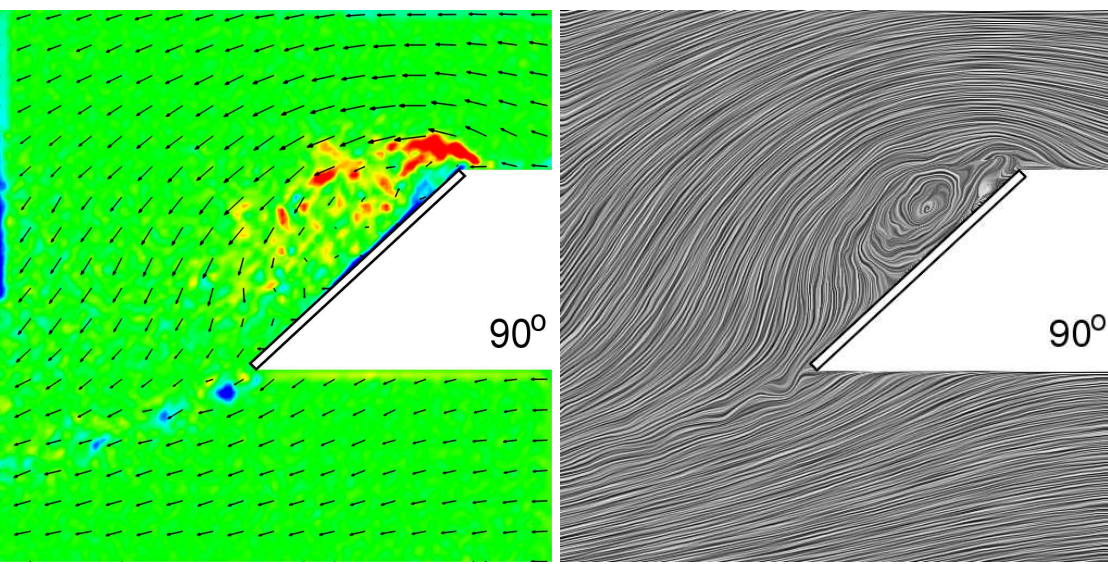

(b) $70 \%$-span
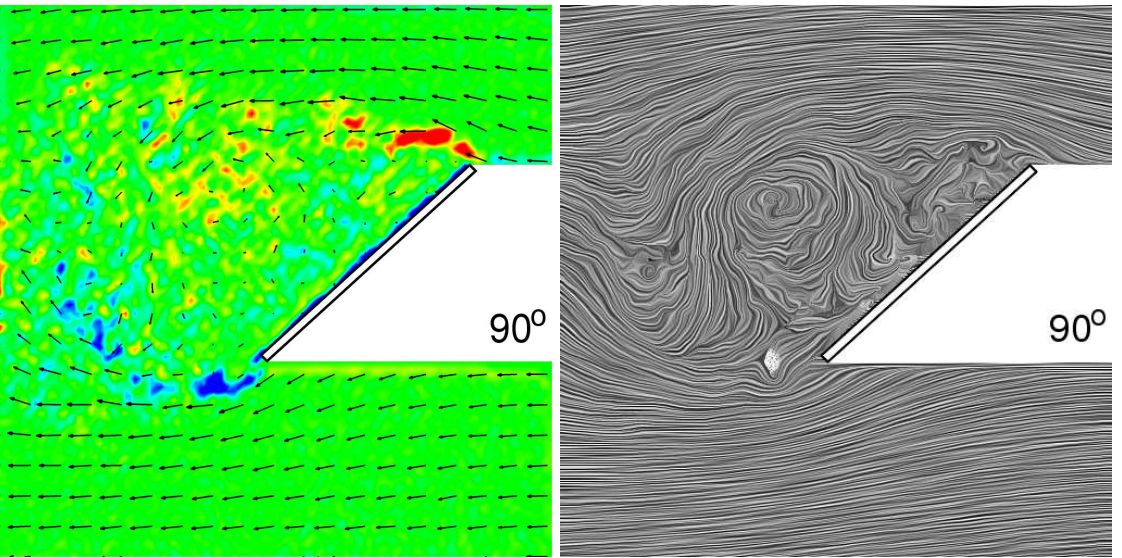

(c) $90 \%$-span

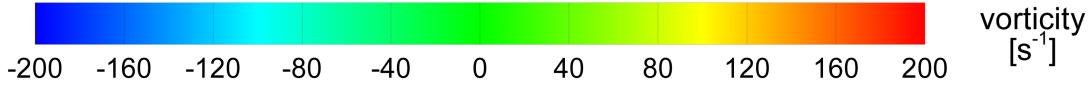

Fig. 13 Effect of spanwise position on chordwise flow for $R e=15000$ at $90^{\circ}$ azimuth, shown by plots of contours of vorticity overlayed with velocity vectors (left) and LIC images (right) 


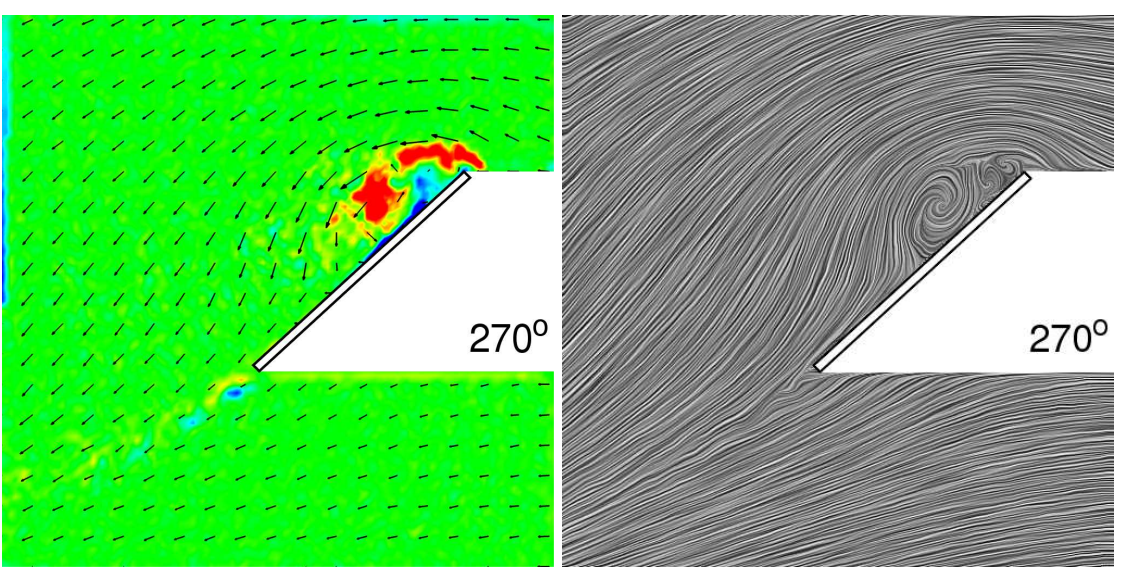

(a) $50 \%$-span
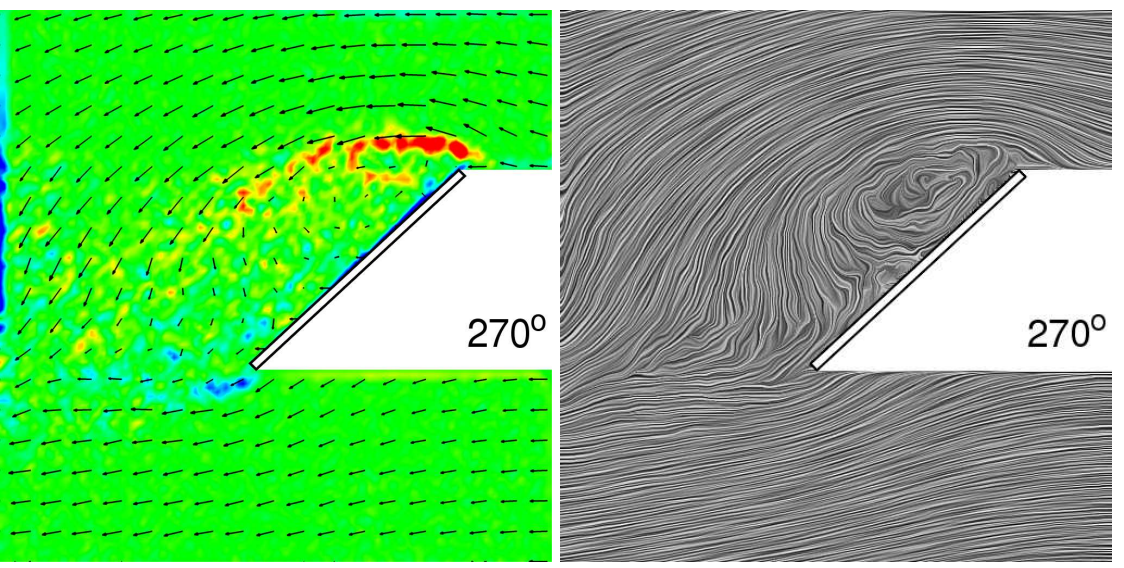

(b) $70 \%$-span
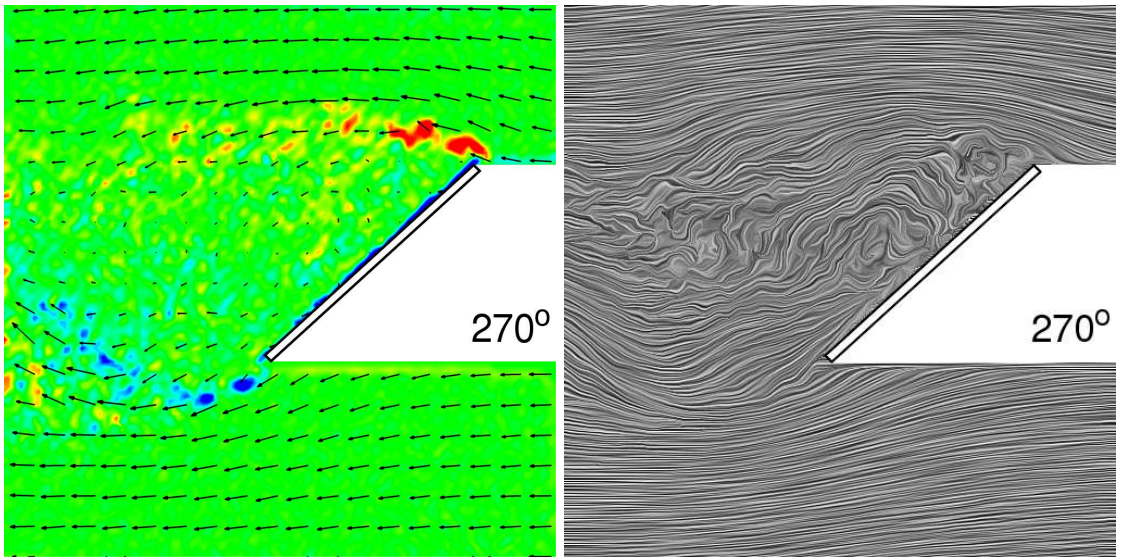

(c) $90 \%$-span

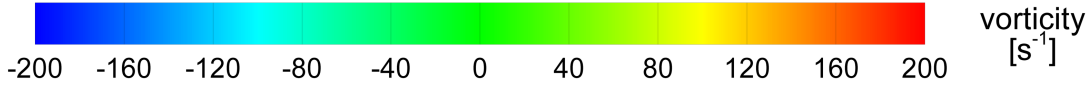

Fig. 14 Effect of spanwise position on chordwise flow for $R e=15000$ at $270^{\circ}$ azimuth, shown by plots of contours of vorticity overlayed with velocity vectors (left) and LIC images (right) 


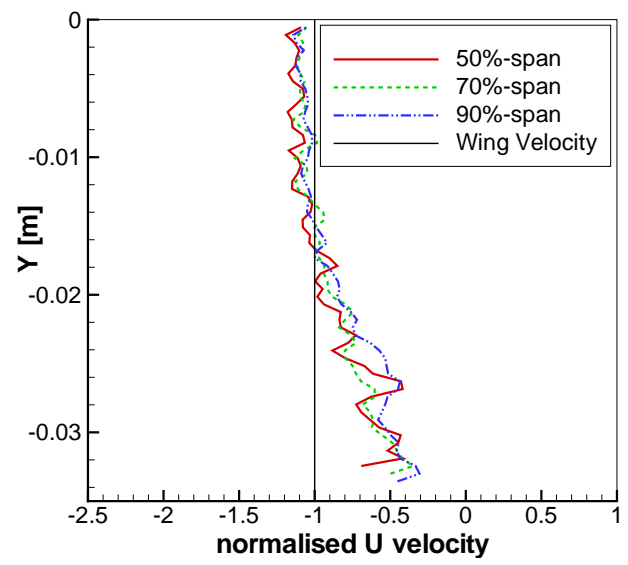

(a) $10^{\circ}$ azimuth

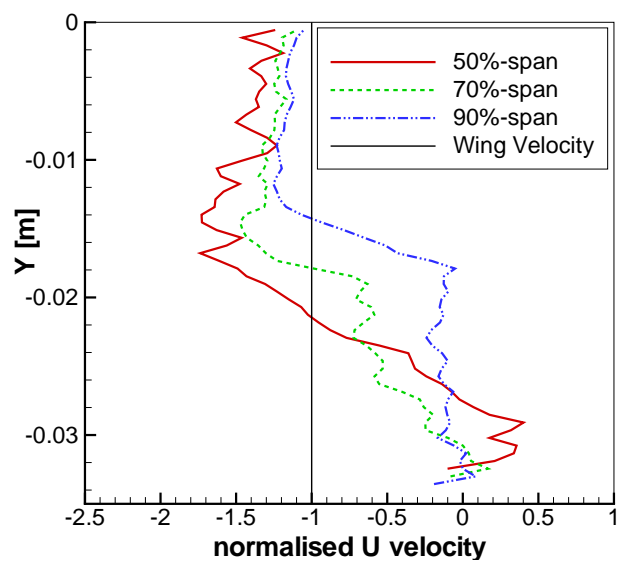

(b) $90^{\circ}$ azimuth

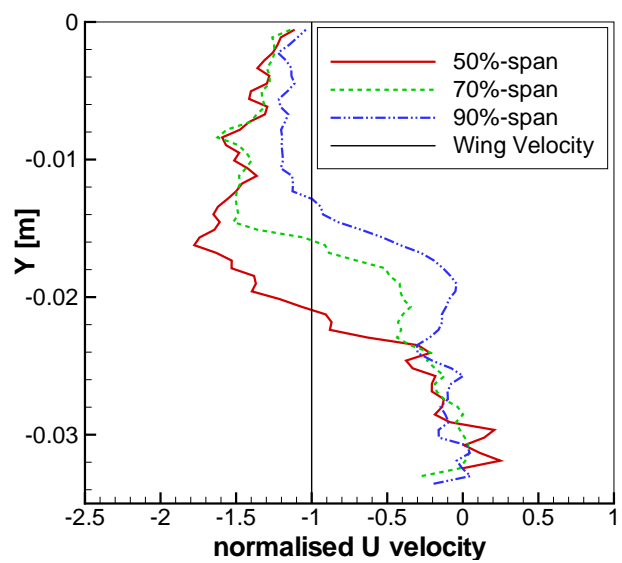

(c) $270^{\circ}$ azimuth

Fig. 15 Effect of spanwise position on chordwise flow for $R e=500$ at azimuths shown $(u$ velocity is normalised w.r.t. local wing velocity; $y$ is measured vertically downwards from the top of the measurement domain to the midchord point on the wing) 


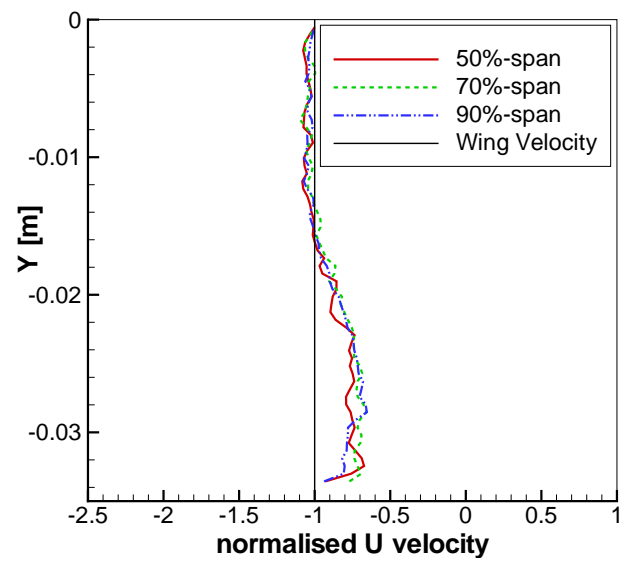

(a) $10^{\circ}$ azimuth

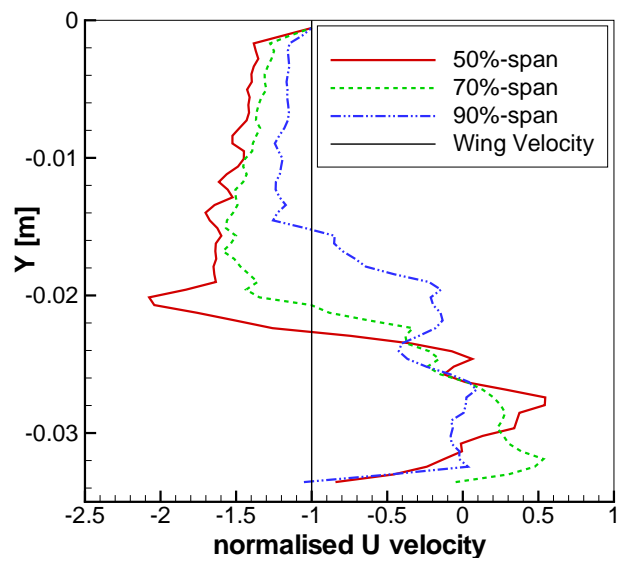

(b) $90^{\circ}$ azimuth

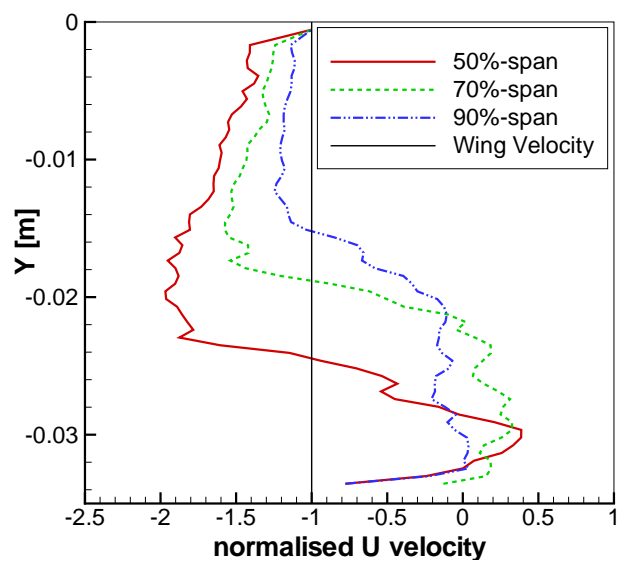

(c) $270^{\circ}$ azimuth

Fig. 16 Effect of spanwise position on chordwise flow for $R e=15000$ at azimuths shown $(u$ velocity is normalised w.r.t. local wing velocity; $y$ is measured vertically downwards from the top of the measurement domain to the midchord point on the wing) 

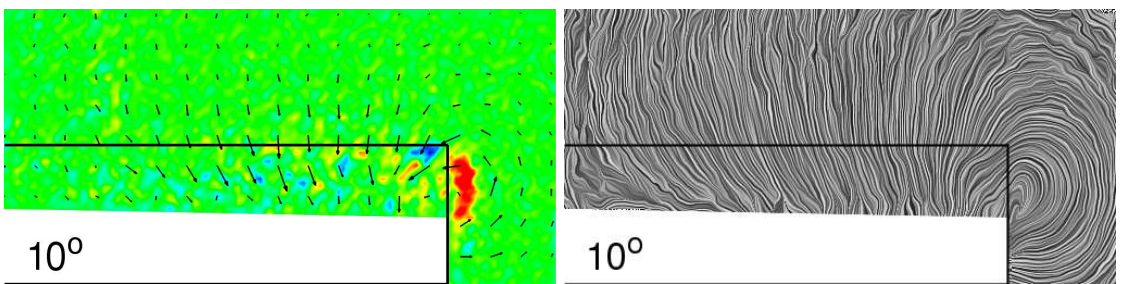

(a) $10^{\circ}$ azimuth

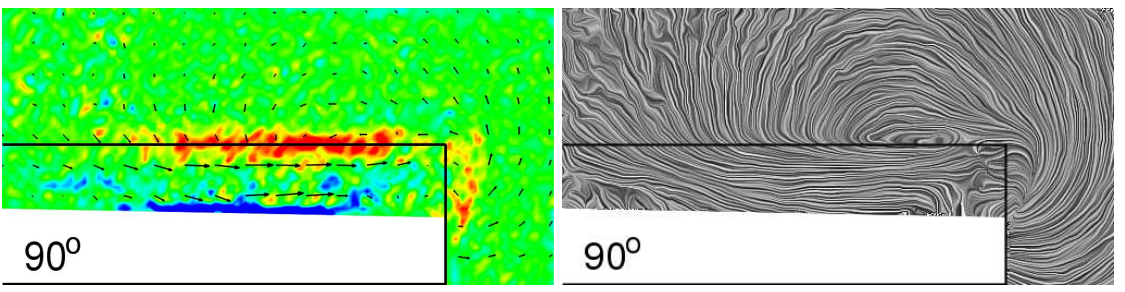

(b) $90^{\circ}$ azimuth
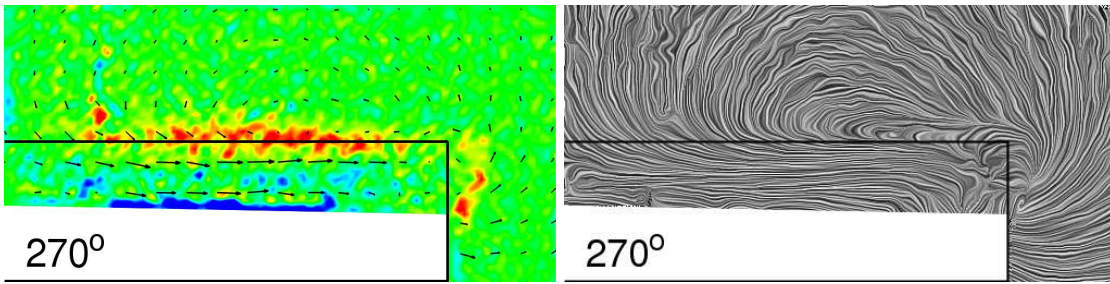

(c) $270^{\circ}$ azimuth

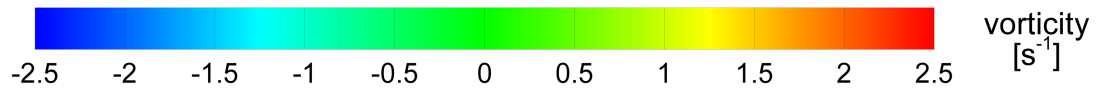

Fig. 17 Effect of azimuthal position on spanwise flow for $R e=500$ at $50 \%$ chord, shown by plots of contours of vorticity overlayed with velocity vectors (left) and LIC images (right); velocity vectors show how flow close to the wing surface changes from chordwise to spanwise

Figures 9 and 12 show that soon after startup (azimuth $\psi=10^{\circ}$ ), the flow field does not differ much at the various spanwise positions. Moving outboard, the leading- and trailing-edge vortices get progressively stronger due to the increasing flow speeds. The leading-edge vortex structure has just begun to form at the 50\%span position, is more developed at the 70\%-span position but then is less tightlyrolled up at the $90 \%$-span position. For the later azimuthal positions $\left(\psi=90^{\circ}\right.$ and $\psi=270^{\circ}$ ), the flow field at the $90 \%$-span position is significantly different from the 50\%- and 70\%-span positions (Figures $10 \& 11$ and Figures $13 \& 14$ ). Whereas the flow rolls up clearly on the leeward side of the wing into the leadingedge vortex for the $50 \%$ - and $70 \%$-span positions, the flow separates at the leading edge for the $90 \%$-span position and interference from the tip vortex is clearly visible. For the later azimuthal positions $\left(\psi=90^{\circ}\right.$ and $\left.\psi=270^{\circ}\right)$, the flow angle at the edges of the measurement domain reduces on moving outboard. 


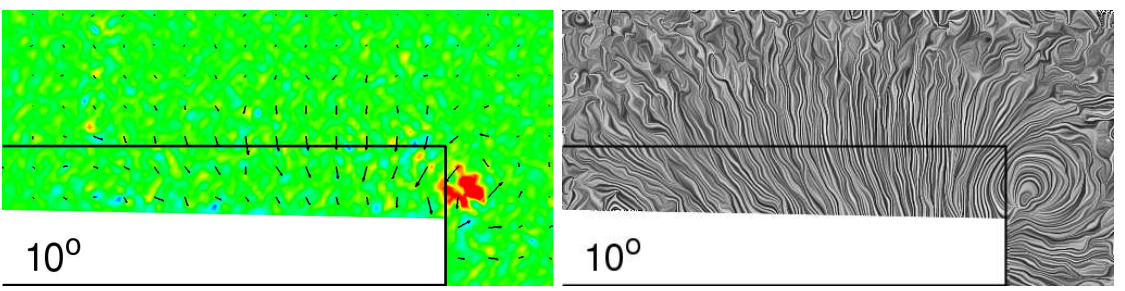

(a) $10^{\circ}$ azimuth

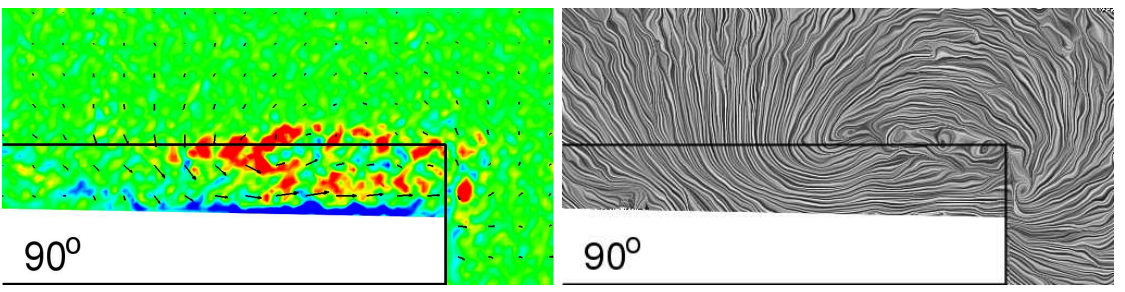

(b) $90^{\circ}$ azimuth

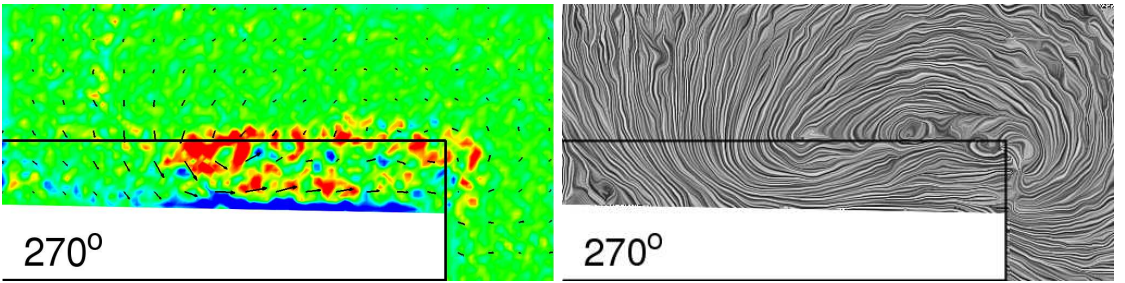

(c) $270^{\circ}$ azimuth

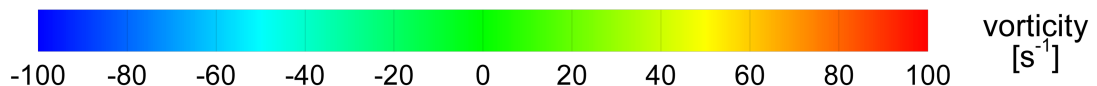

Fig. 18 Effect of azimuthal position on spanwise flow for $R e=15000$ at $50 \%$ chord, shown by plots of contours of vorticity overlayed with velocity vectors (left) and LIC images (right); velocity vectors show how flow close to the wing surface changes from chordwise to spanwise

The effect of spanwise position on the flow field can also be studied using the line plots shown in Figures 15 and 16. These graphs show the horizontal velocity profile (normalised w.r.t. the local wing velocity) through a vertical cross-section taken at the midchord position of the wing. Only data for the flow above the wing are shown. Figures 15 and 16 correspond to Figures $9-11(R e=500)$ and Figures $12-14(R e=15000)$, respectively.

As noted above, in the period immediately following startup $\left(\psi=10^{\circ}\right)$, there is little difference in the flow field between the various spanwise positions. However, as wing sweep proceeds, the size of the leading-edge vortex structure at the $50 \%$-span position is noticeably smaller than at the $70 \%$ - and $90 \%$-span positions (Figures 15(b) \& 15(c) and Figures 16(b) \& 16(c), respectively). Further, the velocity profile for the $90 \%$-span position generally lies between the $50 \%$ - and $70 \%$-span positions showing that the effect of the interference from the tip vortex restricts its size in the outboard regions of the wing. 
The effect of spanwise position on the flow field can also be studied in terms of the spanwise flow above the wing at these positions. This is shown in Figures 17 and 18 for $\operatorname{Re}=500$ and $R e=15000$, respectively. Immediately after startup, most of the flow velocity appears to be chordwise with inboard sections having a greater outboard spanwise component (cf. Figures 17(a) and 18(a)). However, as wing sweep proceeds, the flow in proximity to the upper wing surface becomes spanwise and is nearly horizontal and directed outboard (Figures 17(b) \& 17(c) and Figures 18(b) \& 18(c)). The interference of the leading-edge vortex with the tip vortex also increases but the flow close to the wing remains spanwise and directed outboard.

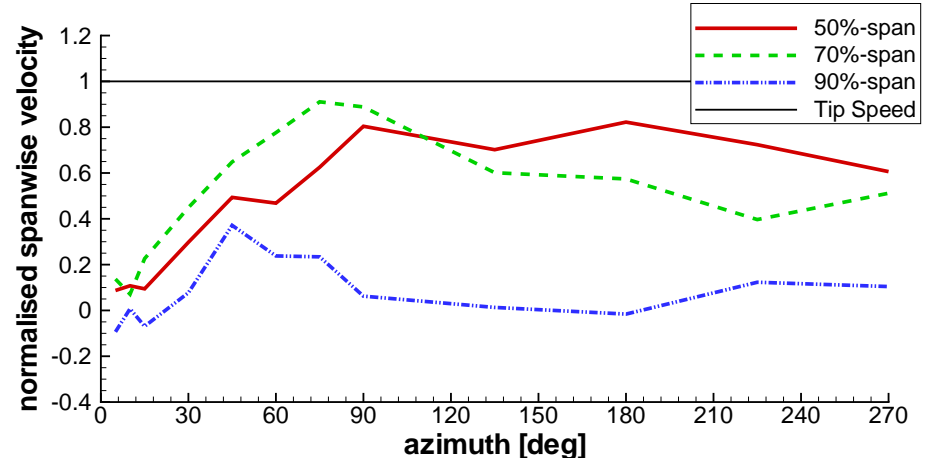

(a) $\operatorname{Re}=500$

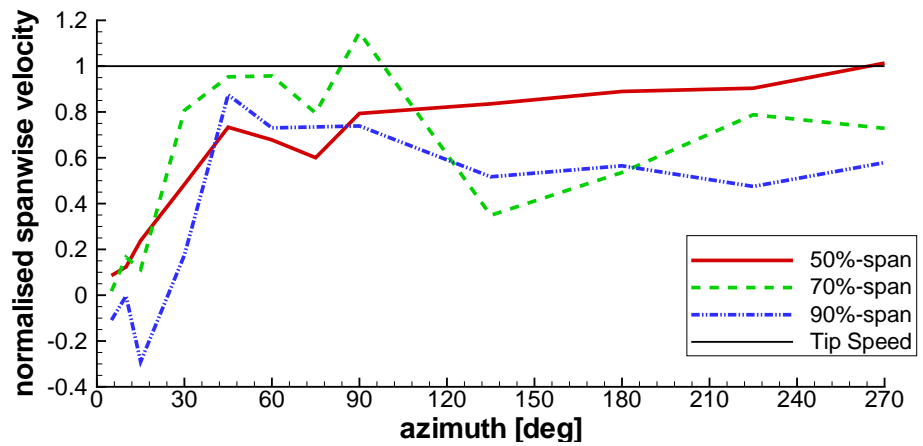

(b) $\operatorname{Re}=15000$

Fig. 19 Plots showing normalised spanwise flow velocity at monitor points in the spanwise measurement plane at $50 \%$-chord for $R e=500$ and $R e=15000$ at various spanwise positions; monitor points are at the vertical level of the wing's quarter-chord point and flow velocities are normalised w.r.t. wingtip speed

To get an idea of the spanwise flow velocities, Figure 19 shows values in the $50 \%$-chord measurement plane at various monitor points along the wing. These points coincide with the chordwise measurement planes used here and are at the vertical level of the wing's quarter-chord point. The spanwise velocity increases 
rapidly after startup, reaches a peak generally around $\psi=60-90^{\circ}$ and then oscillates in the region of $75-85 \%$ of the wingtip velocity. Our results appear to be in agreement with those of Ramasamy and Leishman (2006) who observed spanwise velocities of the order of wingtip speed in their experiments on a model flapping wing. The current experimental results also show that flow velocity magnitudes in the chordwise and spanwise measurement planes are comparable.

\subsection{Effect of Reynolds Number}

The effect of Reynolds number on the flow field is studied in terms of the chordwise (Figures 20 and 21) and spanwise (Figures 24 and 25) flow fields at $\psi=30^{\circ}$ and $\psi=90^{\circ}$, respectively, and the velocity profiles through the leading-edge vortex and the surrounding flow (Figure 23) for the two Reynolds numbers $\operatorname{Re}=500$ and $R e=15000$. Some of the plots discussed above are also used for this comparison (Figures $9-11,15 \& 17$ for $R e=500$ and Figures $12-14,16 \& 18$ for $\operatorname{Re}=15000)$.

From Figures 20 and 21, it can be seen that although the size of the leadingedge vortex appears to increase with Reynolds number, its structure becomes less coherent. This feature can also be seen by comparing Figures 9-11 for $R e=500$ with Figures $12-14$ for $R e=15000$. Close examination of these figures also shows secondary separation near the leading edge in the outboard sections of the wing for $R e=15000$ but not for the lower $R e=500$. This is corroborated by the CFD calculations of Wilkins and co-workers Wilkins et al (2006); Wilkins and Knowles (2007) (see Figure 22).

Another flow feature that is evident from Figures 20 and 21 is the presence of Kelvin-Helmholtz instability at the higher Reynolds numbers. In the discussion in Section 1.2, it was noted that the onset of Kelvin-Helmholtz instability is promoted by higher Reynolds numbers and this has been shown in the experiments carried out here. This result was also predicted by the CFD calculations of (Wilkins and Knowles, 2007) (full details in Wilkins (2008)).

The effect of Reynolds number on the flow features can also be analysed by considering the horizontal velocity profile through the leading-edge vortex. This is plotted for two azimuths $\left(\psi=30^{\circ}, 90^{\circ}\right)$ in Figure 23. Whereas the shapes of the velocity profiles are generally similar, the velocity gradients (and hence, vorticities) are higher for $\operatorname{Re}=15000$ than for $\operatorname{Re}=500$. As noted earlier (see Section 1.2), one of the flow features promoting Kelvin-Helmholtz instability in a fluid is high tangential velocity gradients, which explains the occurrence of Kelvin-Helmholtz instability at the higher Reynolds number in our experiments.

The spanwise flow fields at the two Reynolds numbers (see Figures 24 and 25) show little difference. The flow is generally spanwise in the outboard direction and the tip vortex can be seen interfering and coalescing with the leading-edge vortex. Figure 19 shows the spanwise velocities for both Reynolds numbers more clearly. Although the normalised (w.r.t. wingtip speed) spanwise flow velocity increases to more or less similar values for both Reynolds numbers $(R e=15000$ values are generally slightly higher than those at $\operatorname{Re}=500)$, this 'peak' is reached earlier $\left(\psi \approx 45-60^{\circ}\right)$ by the flow at $\operatorname{Re}=15000$ than at $\operatorname{Re}=500\left(\psi \approx 75-90^{\circ}\right)$. This indicates that the spanwise pressure gradient grows more rapidly at the higher speed. 

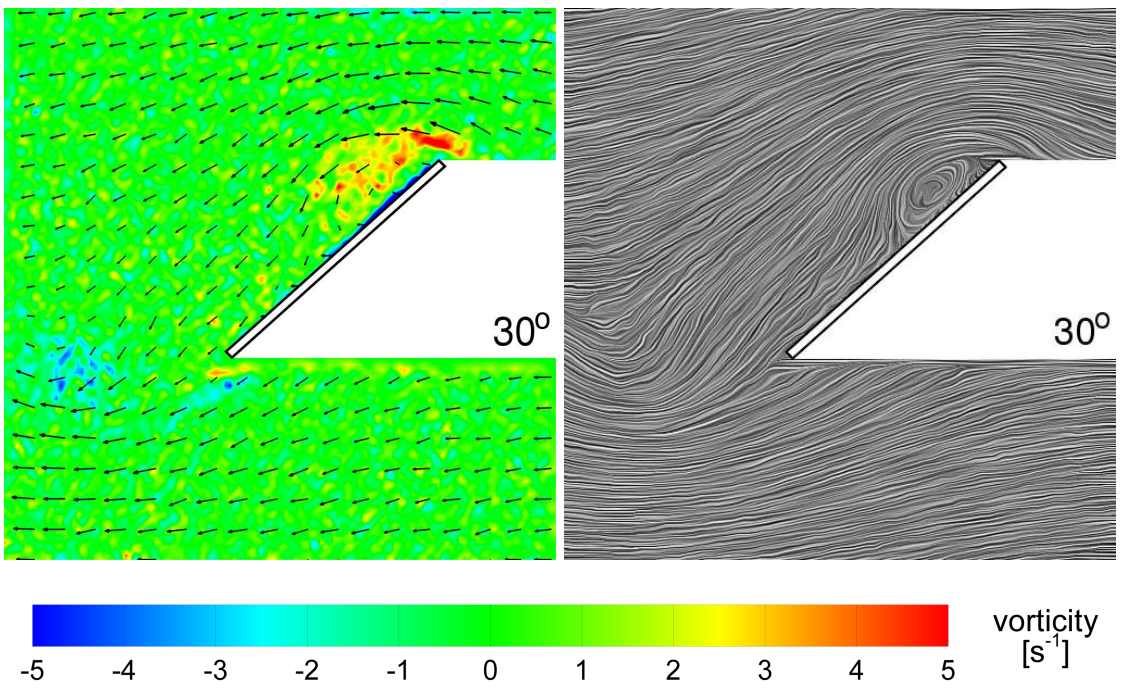

vorticity

(a) $\mathrm{Re}=500$
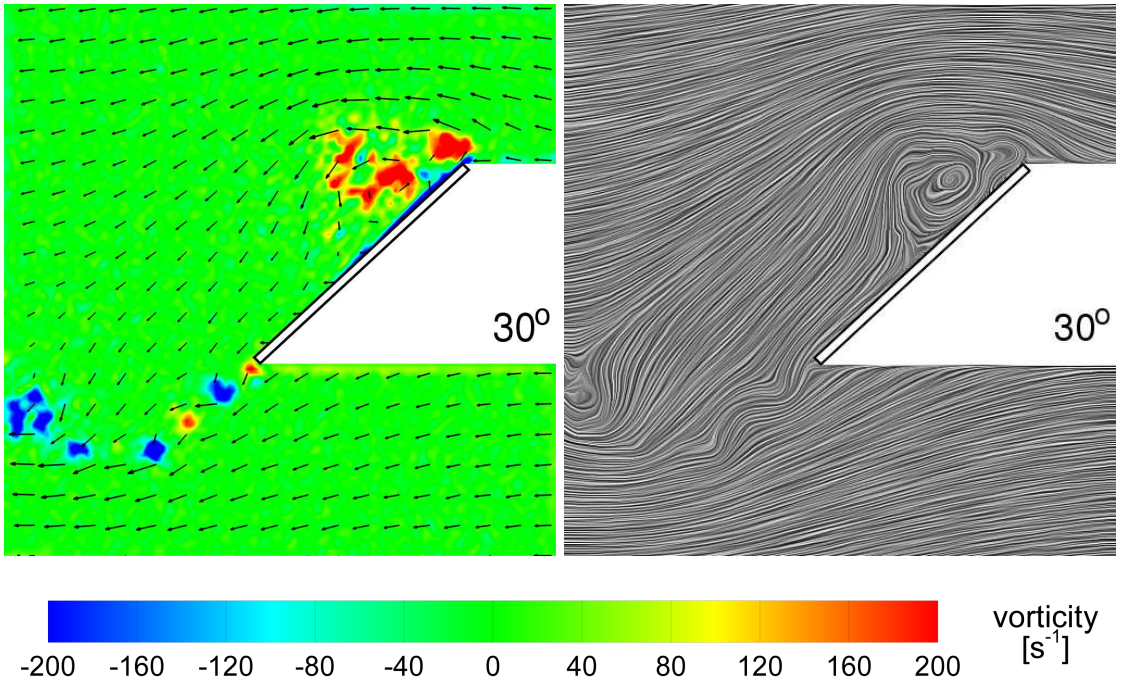

vorticity

(b) $\operatorname{Re}=15000$

Fig. 20 Effect of Reynolds number on chordwise flow at 50\%-span position for $30^{\circ}$ azimuth, shown by plots of contours of vorticity overlayed with velocity vectors (left) and LIC images (right); Kelvin-Helmholtz instability is clearly visible at $R e=15000$ in the bunching together of the trailing-edge wake into smaller vortices 

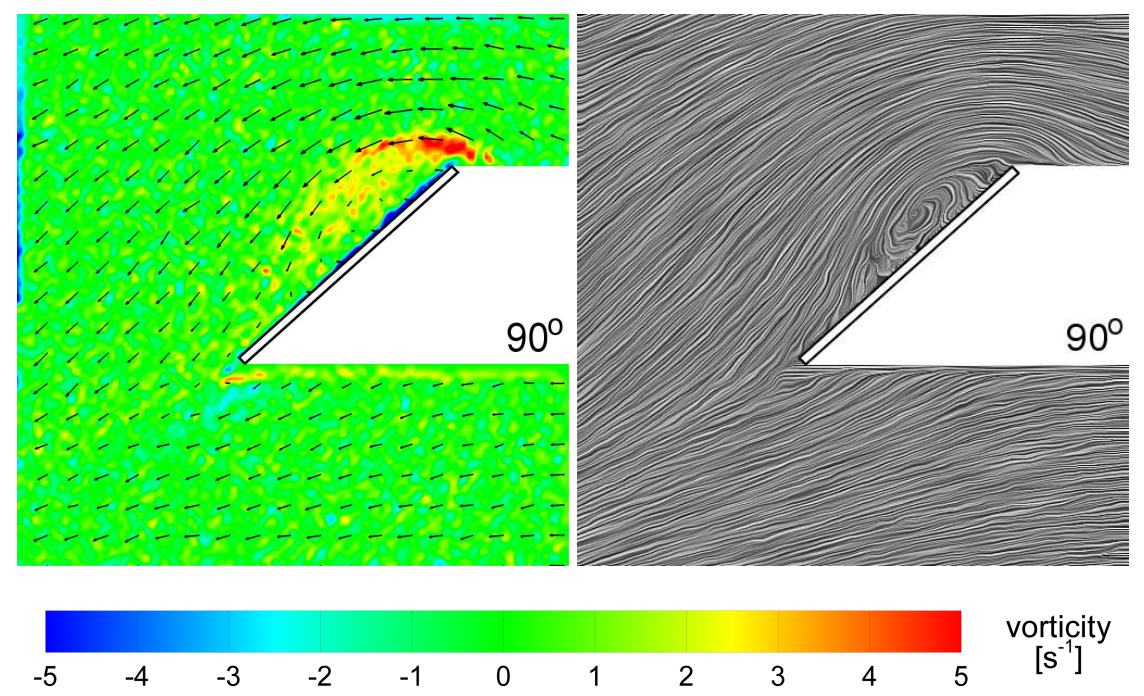

$\underset{\left[s^{-1}\right]}{\operatorname{vortity}}$

(a) $\operatorname{Re}=500$
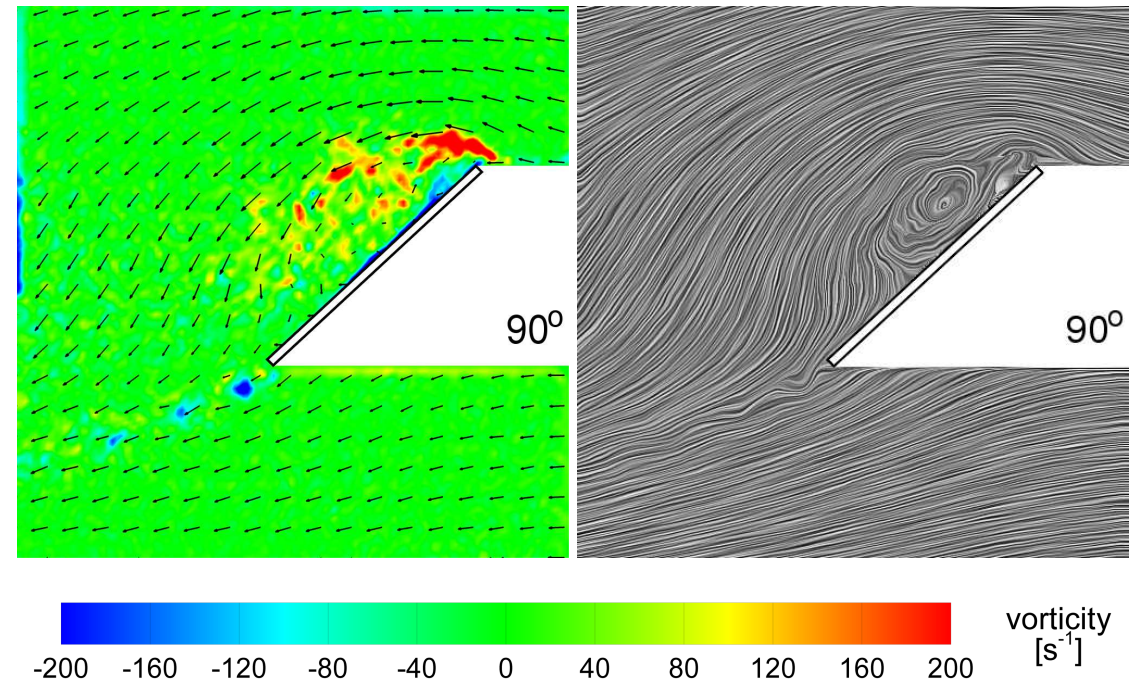

vorticity

(b) $\operatorname{Re}=15000$

Fig. 21 Effect of Reynolds number on chordwise flow at 50\%-span position for $90^{\circ}$ azimuth, shown by plots of contours of vorticity overlayed with velocity vectors (left) and LIC images (right); Kelvin-Helmholtz instability is clearly visible at $R e=15000$ in the bunching together of the trailing-edge wake into smaller vortices 

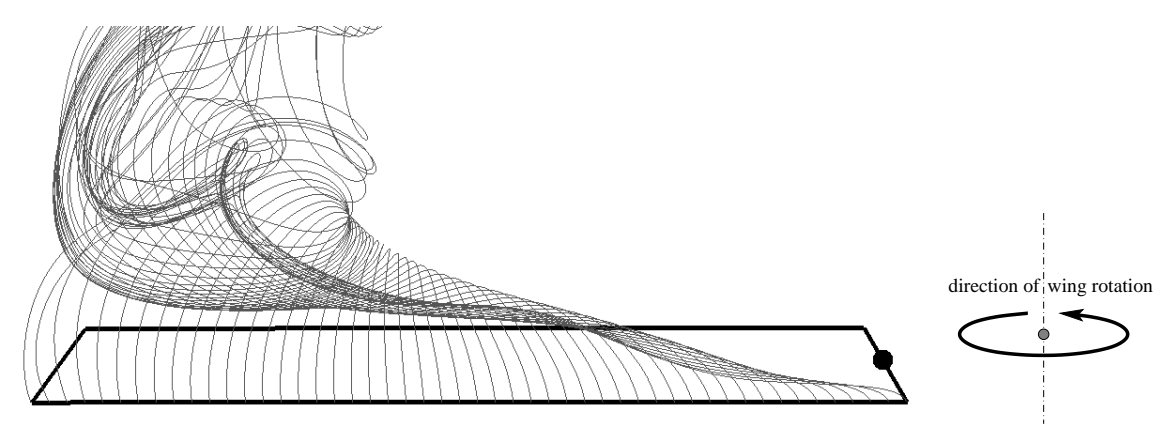

(a) $\operatorname{Re}=500$
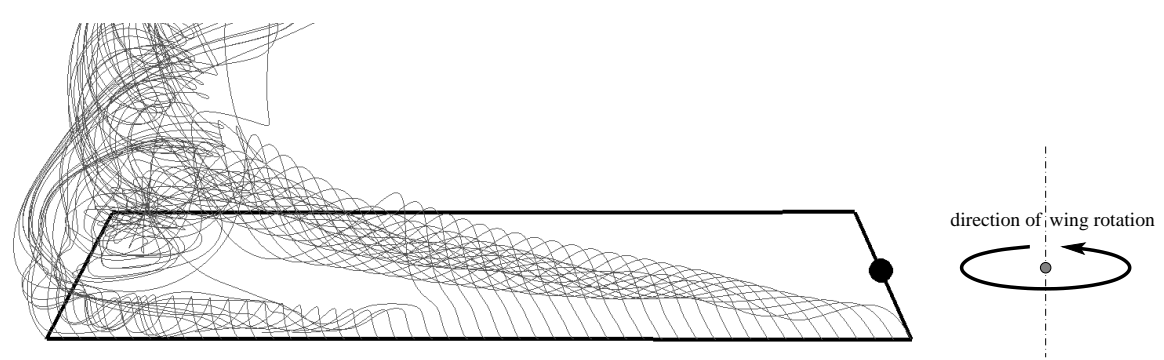

(b) $\operatorname{Re}=15000$

Fig. 22 CFD results of Wilkins et al (2006) showing the effect of Reynolds number. The images show perspective views of the upper surface of the propeller wing with particle streaklines released from points along the leading edge providing flow visulisation; centre of rotation is indicated by the $\bullet$ symbol

At $\psi=30^{\circ}$, three vortex cores (one clockwise core sandwiched between two counterclockwise cores) can be seen in the wing tip region (see Figure 24). The left-hand counterclockwise vortex cores originates from the leading-edge vortex 'turning' through $90^{\circ}$ while the right-hand one is the tip vortex. The fluid 'trapped' between the two cores is forced to move upwards on the left and downwards on the right, resulting in a sandwiched clockwise vortex (see Figure 26). For $\psi=90^{\circ}$ onwards, the two counterclockwise vortex cores coalesce into one large vortex and this condition remains till the end of the experiment at $\psi=270^{\circ}$. This flow feature is less pronounced at $R \mathrm{e}=500$ (see Figure 17).

On the whole, however, the flow field at $R e=15000$ shows similar trends to that of $R e=500$, most likely because the wing has travelled the same number of chord lengths. This characteristic was also highlighted by Ansari and co-workers (Ansari et al, 2006a,b,c).

\section{Conclusions}

From the experimental study presented above, a number of important inferences can be drawn. The results have shown that the leading-edge vortex is generally larger for $R e=15000$ than it is for $R e=500$. The vorticity plots indicate that 


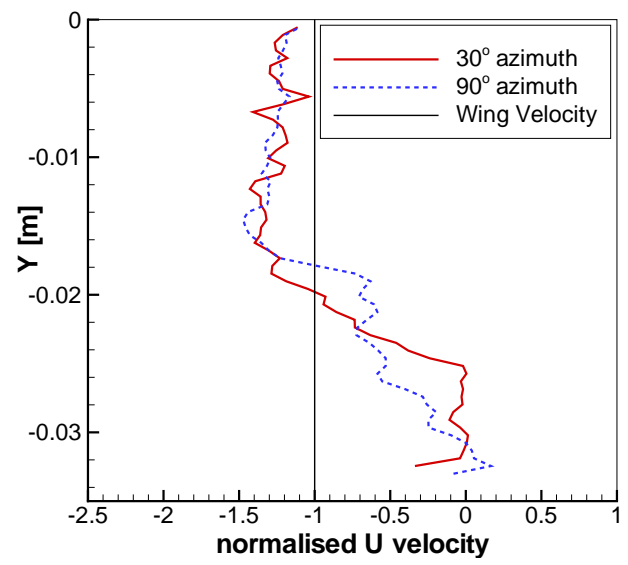

(a) $\operatorname{Re}=500$

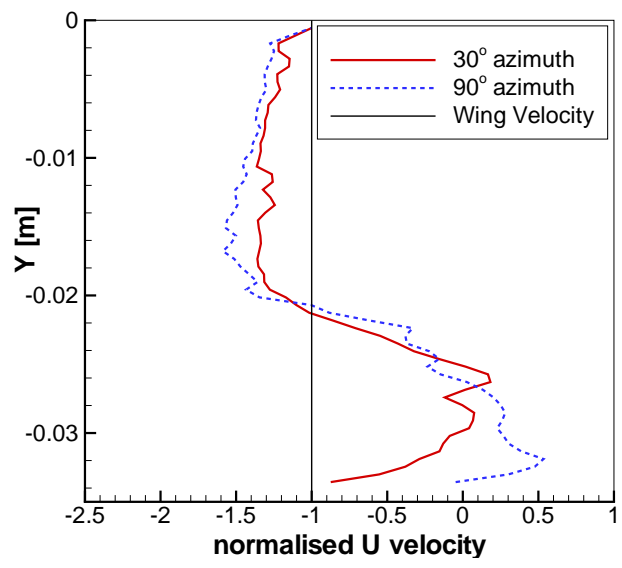

(b) $\operatorname{Re}=15000$

Fig. 23 Effect of Reynolds number at 50\%-span position ( $u$ velocity is normalised w.r.t. local wing velocity; $y$ is measured vertically downwards from the top of the measurement domain to the midchord point on the wing)

the strength of the leading-edge vortex is about 40 times greater for the same comparison, where the Reynolds number ratio is only 30 .

Another important observation is the occurrence of Kelvin-Helmholtz instability. Whereas this appears to be absent for the lower Reynolds number studied here $(\operatorname{Re}=500)$, it is prominent at $R e=15000$. This supports the computational fluid dynamics results of Wilkins and Knowles (2007).

Figures 17 and 18 show, for both Reynolds numbers, the presence of spanwise flow along the wing from the root to the tip. In fact, the spanwise flow appears to be almost more pronounced for the lower Re. Experiments by Dickinson and coworkers (Birch and Dickinson, 2001; Sane and Dickinson, 2001; Birch et al, 2004) revealed the presence of a strong bound leading-edge vortex but they reported 

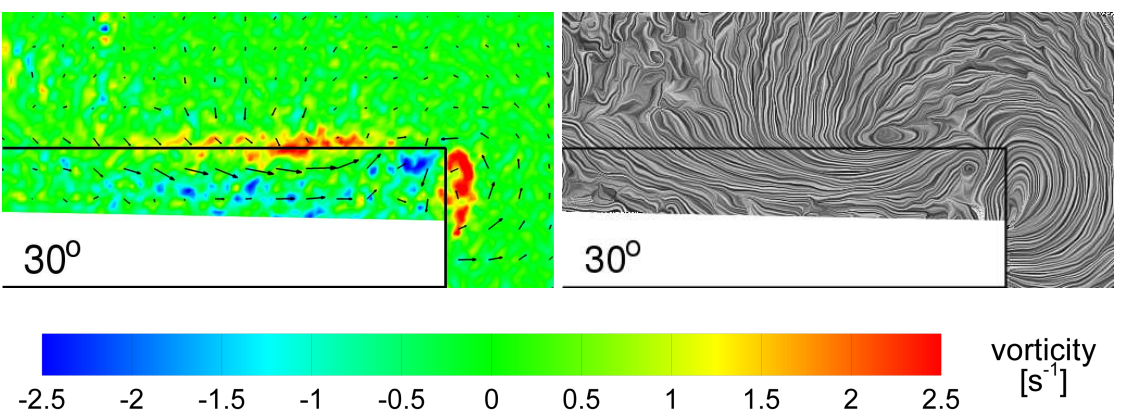

(a) $\operatorname{Re}=500$
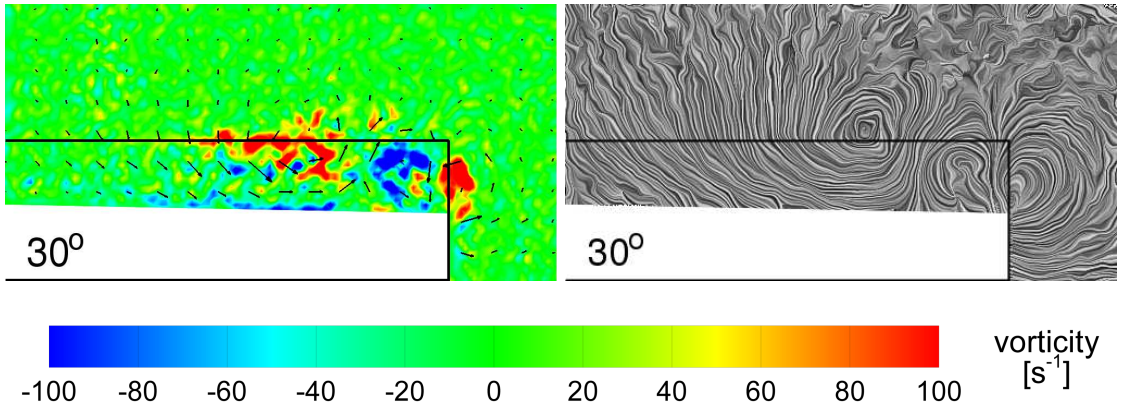

(b) $\operatorname{Re}=15000$

Fig. 24 Effect of Reynolds number on spanwise flow at 50\%-chord position for $30^{\circ}$ azimuth, shown by plots of contours of vorticity overlayed with velocity vectors (left) and LIC images (right); spanwise flow appears to be more prominent for $\operatorname{Re}=500$

only weak spanwise spiralling, prompting them to conclude that the precise flow structure of the leading-edge vortex depends critically on Reynolds number (Birch et al, 2004). This is supported by Ellington (2006) who suggested that spanwise flow exists at all relevant speeds but its spiralling nature becomes less discernable as Reynolds number decreases. Unfortunately, without stereoscopic or volumetric $\mathrm{PIV}$, it is difficult to assess fully the spiralling nature of the leading-edge vortex.

In moving outboard along the wing, the strength of the leading-edge vortex generally increases until it coalesces with the tip vortex whereupon the chordwise flow appears to breakdown. The leading-edge vortex structure is most coherent at the 50\%- and 70\%-span positions; at the 30\%-span position it is very weak due to the low speeds and at the $90 \%$-position, it has broken down due to interference from the tip vortex. Although most of the flow over the wing in the leading-edge vortex is chordwise at startup, this changes rapidly to spanwise (more so for the higher Reynolds number) and spanwise flow velocities on the order of about $80 \%$ of the wingtip speed are maintained thereafter (again, generally higher for the higher Reynolds number).

By comparing the vorticity plots for the chordwise and spanwise results, it can be seen that vorticity levels are about twice as high in the former compared to the latter (e.g. cf. Figure 10(a) and Figure 17(b)). This shows that more vorticity 


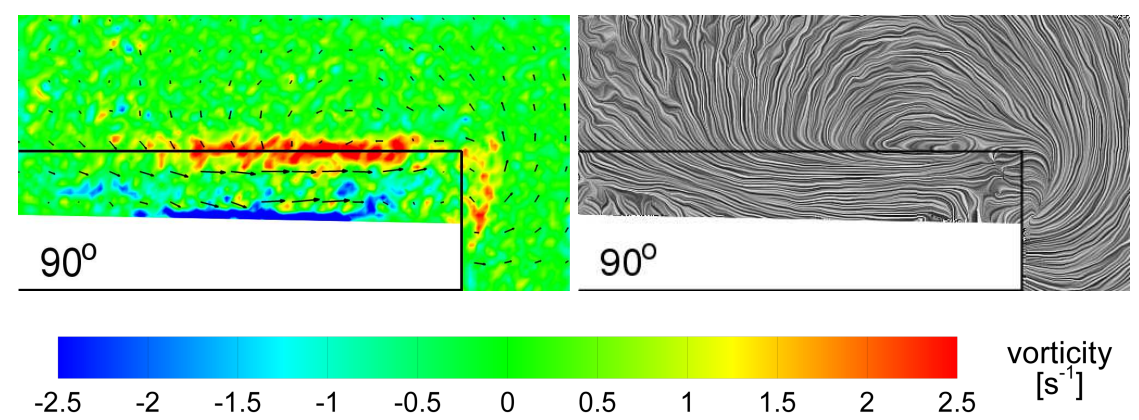

(a) $\operatorname{Re}=500$
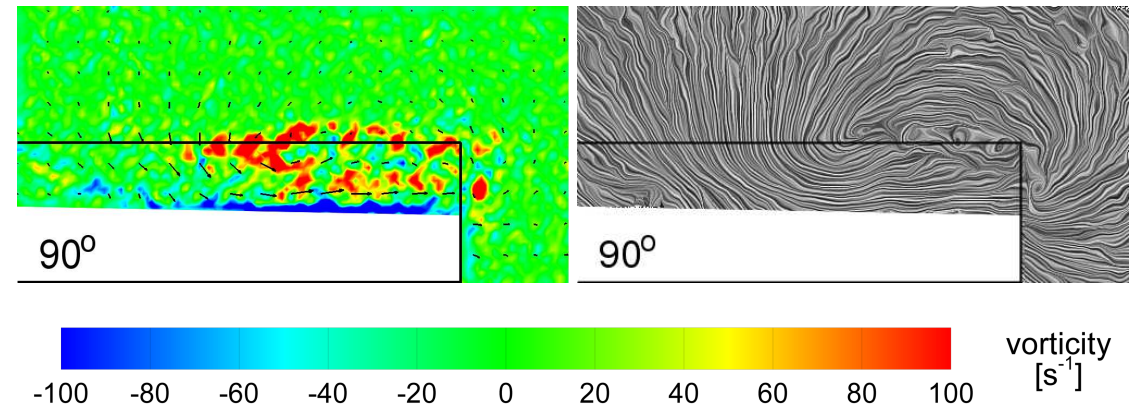

(b) $\operatorname{Re}=15000$

Fig. 25 Effect of Reynolds number on spanwise flow at 50\%-chord position for $90^{\circ}$ azimuth, shown by plots of contours of vorticity overlayed with velocity vectors (left) and LIC images (right); spanwise flow appears to be more prominent for $\mathrm{Re}=500$

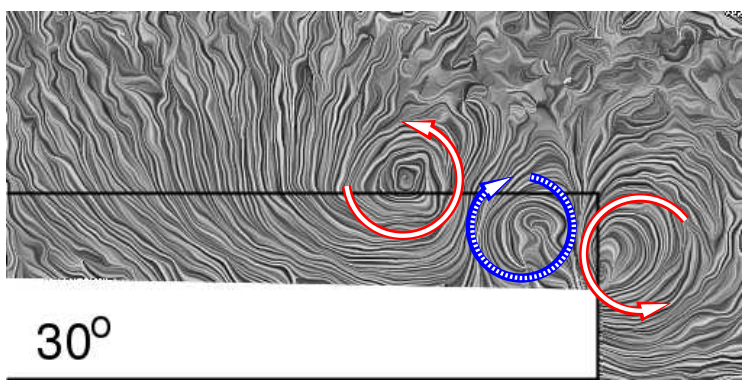

Fig. 26 LIC image showing how a third 'vortex' is formed by virtue of being sandwiched between two vortex cores in the 50\%-chord spanwise measurement plane at $\operatorname{Re}=500$ and $30^{\circ}$ azimuth

is generated in the leading-edge vortex than in the coalesced leading-edge vortex/tip vortex, probably due to destructive interference between the leading-edge vortex and the tip vortex, and energy dissipation in the process. This result is in contrast to low angle-of-attack attached flow where the dominant vorticity comes from the tip vortex. The flow velocity components in the chordwise and spanwise planes are, however, of comparable magnitudes. 
In terms of future work, it will be highly useful to couple the flow field measurements obtained here with force data to relate flow features to the forces and moments they produce, and enhance understanding of the underlying flow structures. The experiments presented here have only permitted the simultaneous measurement of 2 velocity components. Stereoscopic or volumetric PIV measurements will produce data for 3 simultaneous velocity components that will enable better flow description and shed light on the elusive characteristics of the leadingedge vortex on insect-like flapping wings.

Acknowledgements The authors wish to thank Dr. Simon Ritchie for his help in setting up the PIV system.

\section{References}

Ansari SA (2004) A nonlinear, unsteady, aerodynamic model for insect-like flapping wings in the hover with micro air vehicle applications. PhD thesis, Cranfield University (Shrivenham)

Ansari SA, Żbikowski R, Knowles K (2006a) Aerodynamic modelling of insectlike flapping flight for micro air vehicles. Progress in Aerospace Sciences 42(2):129-172, DOI 10.1016/j.paerosci.2006.07.001

Ansari SA, Żbikowski R, Knowles K (2006b) Non-linear unsteady aerodynamic model for insect-like flapping wings in the hover. Part 1: methodology and analysis. Proceedings of the Institution of Mechanical Engineering, Part G: Journal of Aerospace Engineering 220(2):61-83, DOI 10.1243/09544100JAERO49

Ansari SA, Żbikowski R, Knowles K (2006c) Non-linear unsteady aerodynamic model for insect-like flapping wings in the hover. Part 2: implementation and validation. Proceedings of the Institution of Mechanical Engineering, Part G: Journal of Aerospace Engineering 220(3):169-186, DOI 10.1243/09544100JAERO50

van den Berg C, Ellington CP (1997a) The three-dimensional leading-edge vortex of a "hovering" model hawkmoth. Philosophical Transactions of the Royal Society of London Series B 352(1351):329-340

van den Berg C, Ellington CP (1997b) The vortex wake of a "hovering" model hawkmoth. Philosophical Transactions of the Royal Society of London Series B 352(1351):317-328

Birch JM, Dickinson MH (2001) Spanwise flow and the attachment of the leadingedge vortex on insect wings. Nature 412(6848):729-733

Birch JM, Dickson WB, Dickinson MH (2004) Force production and flow structure of the leading edge vortex on flapping wings at high and low Reynolds numbers. Journal of Experimental Biology 207:1063-1072, DOI 10.1242/jeb.00848

Brodsky AK (1991) Vortex formation in the tethered flight of the peacock butterfly Inachis io 1. (lepidoptera, nymphalidae) and some aspects of insect flight evolution. Journal of Experimental Biology 161:77-95

Dickinson MH (1996) Unsteady mechanisms of force generation in aquatic and aerial locomotion. Amer Zool 36:537-554 
Dickinson MH, Götz KG (1993) Unsteady aerodynamic performance of model wings at low Reynolds numbers. Journal of Experimental Biology 174:45-64

Drazin PG, Reid WH (1981) Hydrodynamic Stability. Cambridge Monographs on Mechanics, Cambridge University Press, Cambridge, UK

Dudley R (1992) Biomechanics (Structures \& Systems): A Practical Approach. Oxford University Press

Ellington CP (1984a) The aerodynamics of hovering insect flight: III. kinematics. Philosophical Transactions of the Royal Society of London Series B 305:41-78

Ellington CP (1984b) The aerodynamics of hovering insect flight: IV. aerodynamic mechanisms. Philosophical Transactions of the Royal Society of London Series B 305:79-113

Ellington CP (1995) Unsteady aerodynamics of insect flight. The Society for Experimental Biology 49:109-129

Ellington CP (2006) Insects versus birds: The great divide. In: 44th AIAA Aerospace Sciences Meeting and Exhibit, AIAA, Reno, NV, AIAA-2006-0035

Ellington CP, van den Berg C, Willmott AP, Thomas ALR (1996) Leading-edge vortices in insect flight. Nature 384:626-630

Graham LJW, Soria J (1994) A study of an inclined cylinder wake using digital particle image velocimetry. In: Proc. 7th Int. Symp. on Applications of Laser Techniques to Fluid Mechanics, Lisbon

Grodnitsky DL, Morozov PP (1993) Vortex formation during tethered flight of functionally and morphologically two-winged insects, including evolutionary considerations on insect flight. Journal of Experimental Biology 182:11-40

Hart D (1998) The elimination of correlation errors in PIV processing. In: 9th International Symposium on Applications of Laser Techniques to Fluid Mechanics, Lisbon, p 10.5

Huang H, Dabiri D, Gharib M (1997) On errors of digital particle image velocimetry. Measurement Science and Technology 8(12):1427-1440

Keane R, Adrian RJ (1992) Theory of cross-correlation analysis of PIV images. Applied Scientific Research 49(3):191-215

Keane RD, Adrian RJ (1990) Optimization of particle image velocimeters. parti i: Double pulsed systems. Measurement Science and Technology 1(11):12021215

Kim SC, Lee JY, Sohn SI (2003) Long time computation of two-dimensional vortex sheet by point vortex method. Journal of the Physical Society of Japan 72:1968-1976

Knowles RD, Finnis MV, Saddington AJ, Knowles K (2006) Planar visualization of vortical flows. Proceedings of the Institution of Mechanical Engineering, Part G: Journal of Aerospace Engineering 220(6):619-627

Martin LJ, Carpenter PW (1977) Flow-visualisation experiments on butterflies in simulated gliding flight. Fortschritte der Zoologie 24(2/3):307-316

Maxworthy T (1979) Experiments on the Weis-Fogh mechanism of lift generation by insects in hovering flight. part 1: Dynamics of the 'fling'. Journal of Fluid Mechanics 93:47-63

McCroskey WJ (1981) The phenomenon of dynamic stall. Technical Memorandum TM-81264, NASA, 1-31

Raffel M, Willert C, Kompenhans J (1998) Particle image velocimetry: a practical guide. Springer 
Ramasamy M, Leishman JG (2006) Phase-locked particle image velocimetry measurements of a flapping wing. Journal of Aircraft 43(6):1867-1875, DOI $10.2514 / 1.21347$

Riddle TW, Wadcock AJ, Tso J, Cummings RM (1999) An experimental analysis of vortex trapping techniques. Transactions of the ASME: Journal of Fluids Engineering 121:555-559

Ronneberger O, Raffel M, Kompenhans J (1998) Advanced evaluation algorithms for standard and dual plane particle image velocimetry. In: 9th International Symposium on Applications of Laser Techniques to Fluid Mechanics, Lisbon, p 10.1

Rossow VJ (1978) Lift enhancement by an externally trapped vortex. Journal of Aircraft 15(9):618-625

Sane SP, Dickinson MH (2001) The control and flight force by a flapping wing: Lift and drag production. Journal of Experimental Biology 204:2607-2626

Shyy W, Liu H (2007) Flapping wings and aerodynamic lift: The role of leadingedge vortices. AIAA Journal 45(12):2817-2819, DOI 10.2514/1.33205

Sunada S, Kawachi K, Watanabe I, Azuma A (1993) Performance of a butterfly in take-off flight. Journal of Experimental Biology 183:249-277

Thomas A (priv. comm.) Zoology Department, Oxford University

Westerweel J (1994) Efficient detection of spurious vectors in particle image velocimetry data. Experiments in Fluids 16:236-247

Westerweel J (1997) Fundamentals of digital particle image velocimetry. Measurement Science and Technology 8:1379-1392

Wilkins P, Knowles K (2007) Investigation of aerodynamics relevant to flappingwing micro air vehicles. In: 18th AIAA Computational Fluid Dynamics Conference, AIAA, Miami, FL, pp 1-13, AIAA-2007-4338

Wilkins P, Knowles K (2008) The leading-edge vortex and aerodynamics of insect-based flapping-wing micro air vehicles. In: International Powered Lift Conference (IPLC 2008), The Royal Aeronautical Society, London, UK, pp 114

Wilkins P, Knowles K, Żbikowski R (2006) CFD investigation of aerodynamics relevant to flapping-wing micro air vehicles. In: European Micro Air Vehicle Conference, EMAV, Braunschweig, Germany

Wilkins PC (2008) Some unsteady aerodynamics relevant to insect-inspired flapping-wing micro air vehicles. PhD thesis, Cranfield University (Shrivenham)

Willert CE, Gharib M (1991) Digital particle image velocimetry. Experiments in Fluids 10:181-193

Willmott AP, Ellington CP, Thomas ALR (1997) Flow visualization and unsteady aerodynamics in the flight of the hawkmoth, Manduca sexta. Philosophical Transactions of the Royal Society of London Series B 352:303-316

Woods MI, Henderson JF, Lock GD (2001) Energy requirements for the flight of micro air vehicles. The Aeronautical Journal 105(1043):135-149, paper No. 2546

Żbikowski R (1999a) Flapping wing autonomous micro air vehicles: Research programme outline. In: Fourteenth International Conference on Unmanned Air Vehicle Systems, vol Supplementary Papers, pp 38.1-38.5 
Żbikowski R (1999b) Flapping wing micro air vehicle: A guided platform for microsensors. In: Royal Aeronautical Society Conference on Nanotechnology and Microengineering for Future Guided Weapons, pp 1.1-1.11

Żbikowski R (2000) Flapping wing technology. In: European Military Rotorcraft Symposium, Shrivenham, UK, pp 1-7

Żbikowski R (2002) On aerodynamic modelling of an insect-like flapping wing in hover for micro air vehicles. Philosophical Transactions of the Royal Society of London Series A 360:273-290

Żbikowski R, Pedersen CB, Ansari SA, Galiński C (2003) Flapping wing micro air vehicles. Lecture Series: Low Reynolds Number Aerodynamics On Aircraft Including Applications In Emerging UAV Technology RTO/AVT 104, von Kármán Institute, Belgium 